\title{
Flow and Turbulent Structures Around Simplified Car Models
}

\author{
D.E. Aljure ${ }^{\mathrm{a}}$, O. Lehmkhul ${ }^{\mathrm{a}, \mathrm{b}}$, I. Rodríguez ${ }^{\mathrm{a}}$, A. Oliva ${ }^{\mathrm{a}, *}$ \\ ${ }^{a}$ Heat and Mass Transfer Technological Center (CTTC), Universitat Politècnica de \\ Catalunya-BarcelonaTech (UPC). ETSEIAT, Colom 11, 08222, Terrassa, Barcelona, \\ Spain. Fax: +34937398920. cttc@cttc.upc.edu \\ ${ }^{b}$ Termo Fluids, S.L. Av. Jaquard, 97 1-E, 08222 Terrassa (Barcelona), Spain. \\ termofluids@termofluids.com
}

\section{Abstract}

External car aerodynamics study has great importance in overall car efficiency and ride stability, being a key element in successful automotive design. The flow over car geometries shows three dimensional and unsteady turbulent characteristics. Additionally, vortex shedding, flow reattachment and recirculation bubbles are also found around the bluff body. These phenomena greatly influence the lift and drag coefficients, which are fundamental for ride stability and energy efficiency, respectively. The aim of the present study is focused on the assessment of different LES models (e.g. VMS or SIGMA models), as well as to show their capabilities of capturing the large scale turbulent flow structures in car-like bodies using relative coarse grids. In order to achieve these objectives, the flow around two model car geometries, the Ahmed and the Asmo cars, is simulated. These generic bluff bodies reproduce the basic fluid dynamics features of real cars. First, the flow over

\footnotetext{
*Corresponding author

Email addresses: davidal@cttc.upc.edu (D.E. Aljure), oriol@cttc.upc.edu (O. Lehmkhul ), ivette@cttc.upc.edu (I. Rodríguez ), oliva@cttc.upc.edu (A. Oliva )
} 
both geometries is studied and compared against experimental results to validate the numerical results. Then, different LES models are used to study the flow in detail and compare the structures found in both geometries. Keywords: Automotive aerodynamics, CFD, challenging LES, Turbulence models

\section{Introduction}

Computational fluid dynamics (CFD) has evolved greatly in the past two decades becoming a vital tool in industrial research, development and investigation. Due to the nature of fluid flows, most practical applications deal with turbulent motion. The modeling of this phenomenon is of importance within the CFD industry where vast resources are invested into its research. Within the different possible applications of the CFD technology come aerodynamics and automotive design. The automotive industry makes great advances every year; engine performance has increased greatly in the past decade, along with weight reduction and safety measures. These advances make aerodynamics more important to ensure high efficiency and vehicle drive stability.

The bodies to be studied in the present paper are the widely studied Ahmed car model with a $25^{\circ}$ angle of the rear slanted surface (Ahmed et al. [1], Krajnović and Davidson [2], Minguez et al. [3], Serre et al. [4], Lehmkuhl et al. [5], among others), and the Asmo car model (Perzon and Davidson [6], Nakashima et al. [7], among others). The Ahmed body car is a semirectangular vehicle with a rounded front and a slanted back. The simplified topology of this model allows easy modeling, meshing and comparisons between experimental and numerical results. As for the Asmo car, it has a 
square back rear, smooth surface, boat tailing and an under body diffuser. This model is characterized by no pressure induced boundary layer separation and low drag coefficient.

The Ahmed car was originally used in the experiments of Ahmed et al. [1]. They concluded that the flow structure in the wake was controlled, for a given Reynolds number, by the inclination of the slanted back. Lienhart et al. [8] performed further experiments with the $25^{\circ}$ and $35^{\circ}$ slant back geometries using a laser Doppler anemometer to make detailed measurements of the velocity profiles around the bodies. Numerous numerical studies have been carried out using the aforementioned geometry. The $25^{\circ}$ and $35^{\circ}$ geometries were used in the 9th ERCOFTAC workshop [9]. Results presented by different groups varied greatly, mainly by insufficient grid resolution and convergence. Hinterberger et al. [10] performed large eddy simulations (LES) for the $25^{\circ}$ Ahmed geometry. The authors used two grids of $8.8 \times 10^{6}$ and $18.5 \times 10^{6}$ control volumes $(\mathrm{CV})$. Flow comparison with the experiments performed by Lienhart et al. [8] revealed that the flow structures were well captured. Kapadia et al. [11] used a Spalart-Allmaras based Detached-Eddy Simulation (DES) to model the flow around the $25^{\circ}$ and $35^{\circ}$ Ahmed car using $2.3 \times 10^{6} \mathrm{CV}, 3.1 \times 10^{6} \mathrm{CV}$ and $4.6 \times 10^{6} \mathrm{CV}$ meshes. The authors compared the Reynolds-Averaged Navier-Stokes (RANS) model, the DES and experimental results concluding that their simulations were not very satisfactory as the flow separations were not correctly predicted. Krajnović and Davidson $[2,12]$, in two papers, performed large eddy simulations at a lower Reynolds number $\left(2 \times 10^{5}\right)$ to decrease the computational requirements. Three grids containing $3.5 \times 10^{6}, 9.6 \times 10^{6}$, and $16.5 \times 10^{6}$ were used, whereas a SIMPLEC 
algorithm solved the velocity-pressure coupling. The authors concluded that the influence of the Reynolds number in the wake, after the separations at sharp edges, is small. Furthermore, flow visualization results, including time averaged and instantaneous data, showed structures not observed in experimental set ups. Minguez et al. [3] performed high-order LES for the $25^{\circ}$ slant back case using a mesh of $21 \times 10^{6} \mathrm{CV}$. They used a spectral vanishing viscosity technique to perform the LES around the geometry. The results, conducted at the Reynolds number $R e=7.68 \times 10^{5}$ previously discarded by Krajnović and Davidson $[2,12]$, showed improvement in the overall flow resolution and allowed the visualization of all relevant structures. Recently, Serre et al. [4] compared different approaches (the Smagorinsky subgrid scale model with a wall-function and a mesh with $18.5 \times 10^{6} \mathrm{CV}$, the dynamic Smagorinsky with near-wall resolution and a mesh with $40 \times 10^{6} \mathrm{CV}$, a LES based on spectral approximations with a $21 \times 10^{6} \mathrm{CV}$ mesh, and a Detached Eddy Simulation (DES) with a $k-\omega$ SST model and a mesh with $23.1 \times 10^{6} \mathrm{CV}$ mesh) for computing the flow over the Ahmed geometry at Reynolds number $R e=7.68 \times 10^{5}$. Advantages and disadvantages of the different methods were addressed in the paper. Lehmkuhl et al. [5] used a coarser mesh to simulate the $25^{\circ}$ slant back case with a $8.32 \times 10^{5} \mathrm{CV}$ mesh, and different LES models. Results from this paper showed the good stability and results a conservative formulation of the governing equations can achieve.

As for the Asmo car, it is a model created by Daimler-Benz in the 90's to investigate low drag bodies in automotive aerodynamics and testing of CFD codes with a geometry not related to the development of Mercedes cars. Wind tunnel experiments were made by both, Daimler-Benz and Volvo. Aronson 
et al. [13] studied the flow in the under body region of the car and the differences in drag different geometries (wheels and diffuser) achieved. Their analysis showed that the rear wheels contributed greatly to the drag coefficient. They also saw a significant reduction in drag when the model was equipped with a rear diffuser. Perzon and Davidson [6] performed stationary simulations using RANS models on a $3.8 \times 10^{5} \mathrm{CV}$ mesh and transient simulations in a $7.6 \times 10^{5} \mathrm{CV}$ mesh using the standard $k-\epsilon$ model and the SZLmodel among others. Results of these simulations showed good agreement in pressure and high over prediction in the drag coefficient. Nakashima et al. [7] simulated the flow over the Asmo car using three tetrahedral meshes of $1.3 \times 10^{6}, 5.5 \times 10^{6}$ and $24 \times 10^{6} \mathrm{CV}$ and both RANS and LES approaches. The LES simulations in this paper showed overall better results over the RANS models. Tsubokura et al. [14] performed LES and RANS simulations on the Asmo car using meshes with $5.5 \times 10^{6}$ and $24.3 \times 10^{6}$. Their study on this geometry was aimed to validate the turbulence models for simulations on more complex geometries concluding at the end that LES is a powerful tool within vehicle aerodynamics. Simulations performed within the Xflow project in vehicle aerodynamics (2010) were carried out using a Reynolds number, based on body length, of $R e=2.7 \times 10^{6}$. They used a LES with a wall- adapting local eddy diffusivity (WALE) model within a Lattice-Boltzmann algorithm and concluded that results for the drag coefficient are highly improved when using LES models.

Several difficulties arise when modeling turbulent flows. RANS models often fail to correctly reproduce flow dynamics, especially in detached flows. On the other hand, LES demand high computational resources when dealing 
with industrial-type high Reynolds flows. In the present paper we aim at modeling the flow past aerodynamic simplified cars by means of large-eddy simulations using relative coarse grids. A second-order symmetry-preserving and conservative formulation is used for the discretization of the governing equations. The properties of the present formulation ensure stability and conservation of the kinetic energy balance even with coarse grids at high Reynolds numbers. Thus, in the present paper challenging large-eddy simulations (CLES) are performed. In these CLES, coarse meshes are used in conjunction with conservative and symmetry preserving numerical schemes to reduce the computational requirements necessary to simulate these cases. The capabilities of CLES for reproducing the complex fluid flow phenomena present in automotive aerodynamics using simplified car models are investigated. Both geometries, i.e. the Ahmed and the Asmo cars, have been widely studied in the literature and flow structures around them are well known. By means of different SGS models, the flow structures obtained using different grids are compared to those identified in experimental and numerical works from the literature. In addition to the mean and instantaneous flows, aerodynamics coefficients are also compared. The performance of the different models for predicting these complex aerodynamics forces in car-like geometries is discussed in detail.

\section{Definition of the case}

\subsection{Geometries and computational domain}

The geometries to be considered are the $25^{\circ}$ slant back Ahmed car shown in figure 1a and the Asmo car depicted in figure 1b. 
Both cases are solved in a rectangular computational domain of $9.1944 \times$ $1.87 \times 1.4 \mathrm{~m}$ shown in figure 2 . The front of the car is located at $2.1014 \mathrm{~m}$ downwind from the inlet boundary. The outlet boundary is at a distance of $6.048 \mathrm{~m}$ for the Ahmed car and of $6.282 \mathrm{~m}$ for the Asmo car, measured from the rear end of the body. To simulate the same case conditions as those measured by Lienhart et al. [8] a 3/4 open wind tunnel is considered in both geometries.

\section{Mathematical and numerical model}

In order to study the flow, the filtered incompressible Navier-Stokes equations are solved:

$$
\begin{aligned}
\frac{\partial \overline{\mathbf{u}}}{\partial t}+(\overline{\mathbf{u}} \cdot \nabla) \overline{\mathbf{u}}-\nu \nabla^{2} \overline{\mathbf{u}}+\rho^{-1} \nabla \bar{p} & =-\nabla \cdot \tau \\
\nabla \cdot \overline{\mathbf{u}} & =0
\end{aligned}
$$

where $\overline{\mathbf{u}}$ is the filtered three-dimensional velocity vector, $\bar{p}$ is the filtered pressure scalar field, $\nu$ stands for kinematic viscosity and $\rho$ for the density of the fluid. $\tau$ corresponds to the subgrid-scale (SGS) stress tensor:

$$
\tau=-2 \nu_{S G S} \bar{S}+(\tau: \mathbf{I}) \mathbf{I} / 3
$$

where $\nu_{S G S}$ is the turbulent or subgrid viscosity and $\bar{S}$ is the filtered rate-of-strain tensor, $\bar{S}=\frac{1}{2}\left[\nabla(\overline{\mathbf{u}})+\nabla^{T}(\overline{\mathbf{u}})\right]$.

To close the formulation, the subgrid-scale viscosity should be modeled. Turbulence modeling is carried out in the present paper by using different SGS models: the wall-adapting local-eddy viscosity, WALE, [16], the WALE

model within a variational multiscale framework, VMS, [17], the singular 
values subgrid model, SIGMA, [18], and a model which uses the invariants ( $q$ and $r$ ) of the filtered strain tensor to model eddy viscosity, QR, [19]. Hereafter, a small description of each model is given.

\subsection{QR model}

This model was proposed by Verstappen [19] responding to the question of damping subfilter scales properly. It is based on the invariants of the rate-of-strain tensor. This eddy viscosity model is fairly poses no greater difficulties than a standard Smagorinsky model.

$$
\begin{aligned}
\nu_{S G S} & =\left(c_{q r} l\right)^{2} \frac{r^{+}}{q} \\
c_{q r} & =\frac{1}{\pi}+\frac{1}{24} \\
q & =\frac{1}{4}|\bar{S}|^{2} \\
r & =-\operatorname{det} \bar{S} \\
|\bar{S}| & =(2 \bar{S}: \bar{S})^{1 / 2}
\end{aligned}
$$

where $c_{q r}$ is the model constant, $q$ and $r$ are invariants of $\bar{S}$. The $r^{+}$ stands for positive values of $r$ invariant, otherwise $r$ is set to zero.

\subsection{SIGMA model}

This model, proposed by Nicoud et al. [18], is a subgrid-scale model derived from the analysis of the singular values of the resolved velocity gradient tensor. 


$$
\begin{aligned}
\nu_{S G S} & =\left(c_{\sigma} l\right)^{2} \frac{\sigma_{3}\left(\sigma_{1}-\sigma_{2}\right)\left(\sigma_{2}-\sigma_{3}\right)}{\sigma_{1}^{2}} \\
\sigma_{i} & =\sqrt{\lambda_{i}} \\
G & =\nabla(\overline{\mathbf{u}})^{T} \nabla(\overline{\mathbf{u}}) \\
\mathcal{I} 2 & =\frac{1}{2}\left(\operatorname{tr}(G)^{2}\right)-\left(\operatorname{tr}\left(G^{2}\right)\right), \quad \mathcal{I} 3=\operatorname{det}(G) \\
\mathcal{I} 1=\operatorname{tr}(G), \quad \mathcal{I} 2 & =\lambda_{1} \lambda_{2}+\lambda_{1} \lambda_{3}+\lambda_{2} \lambda_{3}, \quad \mathcal{I} 3=\lambda_{1} \lambda_{2} \lambda_{3}
\end{aligned}
$$

where $c_{\sigma}$ is the model constant, here $c_{\sigma}=1.35, G$ is a symmetric semidefinite positive tensor derived from the velocity gradient tensor, $\lambda_{i}$ are the eigenvalues of the $G$ tensor, and $\sigma_{i}$ are the singular values of the velocity gradient tensor.

\subsection{WALE model}

The WALE model was proposed by Nicoud and Ducros [16] based on the square of the velocity gradient tensor. This SGS model accounts for the effects of the strain and rotation rates, as well as, an appropriate near wall scaling for the eddy viscosity (i.e. $\nu_{S G S} \propto y^{3}$ ).

$$
\begin{aligned}
\nu_{S G S} & =\left(c_{\text {wale }} l\right)^{2} \frac{(\overline{\mathcal{V}}: \overline{\mathcal{V}})^{3 / 2}}{(\bar{S}: \bar{S})^{5 / 2}+(\overline{\mathcal{V}}: \overline{\mathcal{V}})^{5 / 4}} \\
\overline{\mathcal{V}} & =\frac{1}{2}\left(\nabla(\overline{\mathbf{u}})^{2}+\nabla^{T}(\overline{\mathbf{u}})^{2}\right)-\frac{1}{3}\left(\nabla(\overline{\mathbf{u}})^{2}: \mathbf{I}\right) \mathbf{I}
\end{aligned}
$$

where $c_{\text {wale }}$ is the model constant, here $c_{\text {wale }}=0.325$, and $\mathbf{I}$ is the identity matrix. 


\subsection{VMS model}

The variational multiscale method was originally formulated for the Smagorinsky model by Hughes et al. [20]. Later, Vreman [21] extended this method to the classical filtering approach in LES. The VMS framework consists in applying a spatial test filter of length $\hat{l}$ to the already filtered scales $(\bar{f})$, separating this way the resolved scales of motion into large $(\hat{f})$ and small $\left(f^{\prime}\right)$ components. The later is used to model the turbulent viscosity.

$$
f^{\prime}=\bar{f}-\hat{f}
$$

Having applied the filter, a governing equation for the large-scale parts of $\overline{\mathbf{u}}$ can be derived,

$$
\frac{\partial \overline{\mathbf{u}}}{\partial t}+(\overline{\mathbf{u}} \cdot \nabla) \overline{\mathbf{u}}-\nu \nabla^{2} \overline{\mathbf{u}}+\rho^{-1} \nabla \bar{p}=-\nabla \cdot \hat{\tau}-\nabla \cdot \tau^{\prime}
$$

where $\hat{\tau}$ is the subgrid large-scale tensor and $\tau^{\prime}$ is the subgrid smallscale term. Neglecting the effect of the large-scales of motion $(\hat{\tau} \approx 0)$, the turbulent viscosity is calculated by using only the small-scales term $\tau^{\prime}$. This is equivalent to the small-small strategy proposed by Hughes et al. [17]. In the present paper, the WALE model is used to close the formulation as in Lehmkuhl et al. [22]. For more details on the present approach see Appendix A.

\subsection{Numerical method}

The governing equations are discretized on a collocated unstructured mesh by means of finite volume techniques. A second-order conservative scheme is used for the spatial discretization [23]. Such schemes preserve the 
symmetry properties of the continuous differential operators and ensure both stability and conservation of the kinetic-energy balance. As was pointed out by R. and Camussi [23], this methodology might be a good starting point for the formulation of SGS models. Thus, good results even at high Reynolds numbers and with coarse grids might be expected. The velocity-pressure coupling is solved by means of a fractional-step algorithm; the temporal discretization for the convective, diffusive and derivative parts of equation (1) was made using a second order self-adaptive scheme [24]; whereas a backward Euler scheme was used for the pressure gradient.

\subsection{Spatial approximation}

Different types of meshes are used to simulate the flow over the model cars. A fine near wall mesh is necessary to correctly solve the boundary layer and the physical phenomena associated with this zone. Thus, a prism layer is appropriate in this area due to the low non-orthogonal corrections required by this type of elements and the relative simplicity to place these kind of control volumes close to the surface. Furthermore, the near wake zone must be refined to correctly predict the flow structures trailing the car. As the flow moves away the surface, the relative importance of flow structures in the force coefficients and velocity profiles diminish and so does the mesh resolution.

The present simulations are focused on the surroundings of the body and the near wake rather in the far wake. Simulations resolving the full wake of the cars may require different type of meshes, thus clustering a great number of CVs in the far wake to correctly resolve all scales of motion in that area. Three meshes are used with each car model, $\sim 5 \times 10^{5}, \sim 1 \times 10^{6}$ and 
Table 1: Mesh parameters.

\begin{tabular}{cccccc}
\hline Model & Num nodes & min length scale $(\mathrm{mm})$ & $y^{+} \min$ & $y^{+} \max$ & $y^{+}$mean \\
\hline \multirow{2}{*}{ Ahmed } & 486091 & 1.545 & 1.210 & 38.05 & 11.82 \\
& 1045294 & 1.243 & 0.524 & 36.27 & 11.67 \\
& 1561209 & 0.847 & 0.401 & 34.30 & 11.50 \\
\hline \multirow{2}{*}{ Asmo } & 753974 & 1.547 & 1.569 & 34.24 & 14.10 \\
& 1988688 & 0.640 & 0.251 & 17.65 & 4.090 \\
& 2799723 & 0.591 & 0.1447 & 13.89 & 3.517 \\
\hline
\end{tabular}

$\sim 1.6 \times 10^{6} \mathrm{CV}$ for the Ahmed car and $\sim 8 \times 10^{5}, \sim 2 \times 10^{6}$ and $\sim 2.8 \times 10^{6}$ $\mathrm{CV}$ for the Asmo car (see table 1 for details). In the table, some relevant information on the meshes used are given. Here, min. length scale refers to the cube root of the smallest volume element in the mesh, $y^{+} \min$, max and mean corresponds to the minimum, maximum and average location of the first control volume around the car, in wall units.

Figure 3a depicts one of the meshes used for the Ahmed car. A hybrid tetra-prism mesh with a prismatic layer mesh to resolve the boundary layer is used. Furthermore, mesh refinement is done in the back of the car to resolve the near wake.

The mesh around the Asmo car has the same basic structure as that used for the Ahmed car (see figure 3b), i.e. a prismatic layer in the surface of the car and mesh refinement to solve the near wake are both considered. 


\subsection{Boundary conditions}

As for the boundary conditions, a constant velocity inlet profile $\mathbf{u}=$ $\left(u_{r e f}, 0,0\right)$ is imposed. The outlet of the domain is modeled using a convective boundary condition. As the computational domain is a 3/4 open wind tunnel, in the top, front and back boundaries (see figure 2) symmetry conditions are imposed. No-slip conditions are set for the bottom and car surfaces. Both cases the Ahmed and the Asmo cars are simulated using the same boundary conditions and $R e=u_{r e f} h_{r e f} / \nu=7.68 \times 10^{5}$ based on the body height.

All simulations are performed using the TermoFluids parallel code [25]. Hybrid tetra-prism unstructured meshes are generated using the Ansys IcemCFD software. METIS [26] software is used for domain partitioning. All simulations are computed on the in-house JFF cluster, which is composed by 128 nodes with 2 AMD Opteron 2350 Quad Core processor linked with an infiniband DDR network and 40 nodes with 2 AMD 6172 CPUs (16 cores by CPU) linked with a QDR inifiniband network. Each core has 2GB RAM.

\section{Results}

\subsection{Numerical analysis}

For obtaining the numerical results presented in this section, simulations are advanced in time until a statistical stationary flow is reached. Then, in order to ensure converged statistics, instantaneous data is integrated over a sufficiently long-time period. This integration time is about $T U=76$ time units $\left(T U=t u_{r e f} / h_{r e f}\right)$. The VMS turbulence model is used in this section.

In figure 4 the results for the averaged stream-wise velocity and its fluctuations along the symmetry plane of the Ahmed car for different sections in the 
slant surface and the near wake are presented. These results are compared to the experimental results reported by Lienhart et al. [8]. Additionally, results from the $16.5 \times 10^{6}$ mesh from Krajnović and Davidson [2] and from the $21 \times 10^{6}$ mesh from Minguez et al. [3] are also plotted for comparison sake. As can be seen in the figure, the mesh of $1 \times 10^{6} \mathrm{CV}$ is capable of reproducing with quite good agreement the experimental results. A further refinement of the mesh $\left(1.6 \times 10^{6} \mathrm{CV}\right)$ does not improve substantially the results, but the computational cost increases. The flow resolution near the boundary layers from Krajnović and Davidson [2] show excellent agreement with experimental results. Results obtained in the present paper, although with much coarser meshes, are comparable in quality as those obtained by previous authors with finer meshes. When comparing results from Minguez et al. [3] with the ones obtained in the present work it is important to observe the recuperation region, i.e. the velocity profiles further away from the wall. In Minguez et al. [3] the velocity magnitudes are under-predicted, in contrast to the flow evolution obtained in the present paper, by Krajnović and Davidson [2] and by Lienhart et al. [8]. The profiles of the stream-wise velocity fluctuations show the same fact the velocity does, the mesh of $\sim 1 \times 10^{5} \mathrm{CV}$ mesh is capable of reproducing with quite good agreement the experimental results and is also comparable to the results by other authors obtained with larger meshes.

Figure 5 shows the average pressure coefficient $\left(c_{p}=\left(p-p_{\infty}\right) /\left(0.5 \rho u_{r e f}^{2}\right)\right)$ profile alongside the symmetry plane of the underbody and in the back of the Asmo car. In this case, the three meshes studied (see table 1) are compared to the experimental results reported by both Volvo and Daimler-Benz (taken 
from [6]) and to the numerical results of Perzon and Davidson [6] and Tsubokura et al. [14]. As can be seen in the figure, the results obtained with the $1.8 \times 10^{6} \mathrm{CV}$ mesh and $2.8 \times 10^{6}$ are quite close to the experimental data in the underbody, especially near the front, where the pressure spike is properly resolved. The rear pressure spike is under predicted. Furthermore, Perzon and Davidson [6] data from transient RANS simulation on $7.6 \times 10^{5}$ cells shows a larger error than present work data. Results by Tsubokura et al. [14] closely resemble the experimental ones, this profile comes from a LES simulation on $24.3 \times 10^{6} \mathrm{CV}$, which is much finer than the meshes used in the present work. Results in the back of the car by Tsubokura et al. [14] show a behavior very close to the experimental measurements, whereas some differences are found for all meshes in the present investigation. Comparing those (Tsubokura et al. [14]) results from a $24.5 \times 10^{6} \mathrm{CV}$ mesh with the present computations with a $2.8 \times 10^{6} \mathrm{CV}$ mesh it is shown that, in spite of the coarse mesh and showing some errors, the resolution of the governing equations is performed rather accurately. When examining table 2 two facts stand out. First, the coarse meshes $\left(5 \times 10^{5}\right.$ and $\left.8 \times 10^{5}\right)$ do not have enough resolution for a proper resolution of the flow. This is especially evident due to the large difference present between the drag values obtained with each SGS model. Second, the finer meshes $\left(1.6 \times 10^{6}\right.$ and $\left.2.8 \times 10^{6}\right)$ show a consistent behavior, the difference between experimental data and the present work is under $10 \%$ for all models with these meshes. Therefore, the behavior of the different turbulence models was evaluated using these meshes. It is worth noting that although using finer meshes increases the quality of the solution, it should borne in mind that the higher the mesh resolution, the larger computational 
Table 2: Pressure drag (Ahmed car: $c_{p}=0.285$ [1]) and drag (Asmo car: $c_{p}=0.153$ [13]) with different meshes and turbulence models. Relative errors [\%] are given in brackets.

\begin{tabular}{cccccc}
\hline Model & Mesh & QR (\% dif) & VMS & SIGMA & WALE \\
\hline Ahmed & $5 \times 10^{5}$ & $0.312(9.47)$ & $-0.137 !(148.1)$ & $0.005(98.25)$ & $0.279(2.11)$ \\
& $1 \times 10^{6}$ & $0.301(5.61)$ & $0.302(5.96)$ & $0.281(1.40)$ & $0.310(8.77)$ \\
& $1.6 \times 10^{6}$ & $0.290(1.75)$ & $0.292(2.46)$ & $0.284(0.35)$ & $0.304(6.67)$ \\
\multicolumn{2}{c}{ Krajnović and Davidson [2] } & & $0.292(2.46)$ & \\
\hline \multirow{2}{*}{ Asmo } & $8 \times 10^{5}$ & $0.169(10.38)$ & $0.139(9.15)$ & $0.160(4.58)$ & $0.143(6.54)$ \\
& $2 \times 10^{6}$ & $0.165(7.78)$ & $0.164(7.19)$ & $0.187(22.48)$ & $0.171(15.55)$ \\
& $2.8 \times 10^{6}$ & $0.153(0.13)$ & $0.154(0.65)$ & $0.168(9.80)$ & $0.162(5.88)$ \\
\multicolumn{2}{l}{ XFLOW $[15]$} & & & $0.151(1.31)$ & \\
\hline
\end{tabular}

resources required.

\subsection{Challenging LES analysis}

One of the main objectives of the present paper is to assess the validity of flow solutions using LES in coarse meshes and different SGS models. The performance of four different models is here analyzed. These models are: the QR, SIGMA, WALE and VMS, all presented in section 2. Hereafter, the results for both geometries on the $1.6 \times 10^{6}$ and $2.8 \times 10^{6} \mathrm{CV}$ meshes for the Ahmed and the Asmo cars, respectively, are presented.

\subsubsection{Ahmed car}

As mentioned before, for this geometry the experimental data obtained by Lienhart et al. [8] is available. In figure 6 , the average stream-wise velocity 
and its fluctuations in the mid plane of the slant wall and the near wake are presented. Results of the stream-wise velocity profiles shown that the WALE and SIGMA models are closer to the experimental data than the other two models. Moreover, it can be seen that the WALE, SIGMA and VMS models yield good results in the boundary layer of the slant wall, where the QR model fails to predict the flow behavior correctly. Results for all models in the back side deviate from the measured values. However, further downstream they recover the agreement with experimental data.

Figure $6 \mathrm{~b}$ shows the stream-wise velocity fluctuations, urms. In this case, the VMS model yielded the best results of all four models. In contrast, the less accurate data is the one resulting from the QR model.

Figure 7 shows the mean velocity and root mean square velocity profiles at the $y=0.1$ plane. Results for the mean stream-wise velocity do not vary much from one model to other, being the closest to the experimental results those obtained with the SIGMA model. This same trend can be observed in figure $7 \mathrm{~b}$, where even though the variations are larger, results are similar for all models. Again, the SIGMA model results are a tad closer to the experimental data.

Two of the most important aerodynamic parameters are the lift and drag forces. The drag coefficient quantifies the advancing resistance posed by the air (surrounding fluid) onto the vehicle. The lift coefficient quantifies the force exerted onto the vehicle in the direction perpendicular to the flow. Reducing the drag coefficient of a vehicle will reduce its overall fuel consumption. On the other hand, reducing the lift coefficient will improve ride stability and cornering performance. 
Table 3: Ahmed car drag coefficient for the different models. Relative errors [\%] are given in brackets.

\begin{tabular}{cccc}
\hline Model & Pressure & Skin friction $\left(\times 10^{3}\right)$ & Total \\
\hline VMS & $0.292(2.46)$ & 9.406 & 0.302 \\
QR & $0.290(1.75)$ & 8.926 & 0.299 \\
WALE & $0.304(6.67)$ & 12.33 & 0.315 \\
SIGMA & $0.284(0.35)$ & 10.82 & 0.294 \\
Exp [1] & 0.285 & & \\
\hline
\end{tabular}

Table 3 shows the drag coefficient obtained with the different SGS models. The SIGMA model performs well in predicting the drag, especially the pressure, whereas the WALE shows the worst results. Furthermore, the VMS and the QR models yield similar results. Indeed all four models perform in a similar fashion when resolving the viscous drag. Regarding lift forces, as can be seen in table 4, the Ahmed geometry has a high lift coefficient, being of the same magnitude as the drag coefficient. All models give close values of the lift coefficient except for the WALE model whose deviations are the largest.

In general, the SIGMA and VMS models outperform the QR and WALE models. SIGMA and VMS results for the mean velocity and and its fluctuations are both very close to the experimental results. However, the VMS model over predicts the drag coefficient. 
Table 4: Ahmed car lift coefficient for the different models

\begin{tabular}{cccc} 
Model & Pressure & Skin friction $\left(\times 10^{5}\right)$ & Total \\
\hline VMS & 0.331 & 12.88 & 0.331 \\
QR & 0.322 & 7.515 & 0.322 \\
WALE & 0.257 & 5.78 & 0.257 \\
SIGMA & 0.314 & 25.53 & 0.315 \\
\hline
\end{tabular}

\subsubsection{Asmo car}

The same models are used to solve the flow over the Asmo car. Figure 8a shows the pressure distribution along the symmetry plane in the underbody. Results obtained with the QR model are the closest to the experimental results, showing very good agreement with those of the Daimler-Benz. The SIGMA, VMS and WALE models show a good prediction of the front pressure spike. However, they fall short from the experimental results in the rear spike. WALE and SIGMA models under-predict the pressure distribution in the frontal part of the car. The behavior of the VMS model is good. In the upwind section the results are close to the Daimler-Benz data, whereas in the downwind section the results closely resemble those of the WALE and SIGMA models.

Figure $8 \mathrm{~b}$ shows the results over the rear of the car. Given the dispersion in the measurements, all models reasonably agree with the experimental results. The SIGMA model shows a good pressure distribution but it slightly under predicts it. The VMS model under-predicts the pressure coefficient near the roof. The QR model shows good agreement with experimental data in the top half of the car and and over predicts the pressure distribution near 
Table 5: Asmo car drag coefficient. Relative errors [\%] in brackets.

\begin{tabular}{cccc}
\hline Model & Pressure & Skin friction & Total \\
\hline VMS & 0.143 & 0.011 & $0.154(0.65)$ \\
QR & 0.140 & 0.013 & $0.153(0.13)$ \\
WALE & 0.151 & 0.013 & $0.162(5.88)$ \\
SIGMA & 0.154 & 0.014 & $0.168(9.80)$ \\
Exp [13] & & & 0.153 \\
\hline
\end{tabular}

the bottom. Results obtained with the WALE model best resemble the experimental measurements. Note that the QR model predicts a high negative pressure spike, probably associated with how spatial discretization affects the model results, especially in the zones where the quality of the mesh is low. This spike is also found in Tsubokura et al. [14] results.

Figure 9a shows the results in the symmetry plane along the roof of the model. Results from all models are close to the experimental data. As will be shown in next section, there is a low pressure bubble wrapping around the car and centered in the middle of the roof. This produces a negative pressure spike in the figure, which all models reproduce in a similar fashion. Finally, figure 9b shows the pressure distribution in the frontal section. All models perform well on this area, with a correct prediction of the stagnation point. The SIGMA model predicts a higher stagnation pressure than the other three, being closer to the Volvo measurements. In general, in both the roof and front parts of the body, all models behave similarly.

Tables 5 and 6 show the force coefficients on the Asmo geometry. As it can be seen, the drag coefficient of the Ahmed car nearly double the drag 
Table 6: Asmo car lift coefficient

\begin{tabular}{cccc}
\hline Model & Pressure & Skin friction $\left(\times 10^{4}\right)$ & Total \\
\hline VMS & -0.034 & 13.01 & -0.023 \\
QR & -0.039 & 16.60 & -0.038 \\
WALE & -0.069 & 8.95 & -0.058 \\
SIGMA & -0.030 & 18.69 & -0.028 \\
\hline
\end{tabular}

coefficient of the Asmo car. The drag coefficients calculated with the VMS and QR models are closer to the experimental value reported by Aronson et al. [13] than the other two models. With this mesh drag is over predicted by the WALE and the SIGMA models. All models predict a negative lift coefficient, the VMS, QR and SIGMA models report a very similar value. Comparing tables 4 and 6 it can be seen that the lift coefficient for the Asmo car is negative (down force) and its magnitude is smaller than the positive lift coefficient found for the Ahmed car.

The QR and WALE models showed better results for the pressure distribution for the Asmo car. The drag coefficients were better predicted by the VMS and QR models.

Flow over the Ahmed car is better solved by the SIGMA and the VMS models. Flow in this geometry is characterized by heavy flow separation and a geometry influenced boundary layer. For the Asmo car, the QR and the VMS models show better results. The flow in this geometry is characterized by a laminarized flow structure and no pressure induced boundary layer separation. The QR model did not perform well in the geometry-influenced boundary layer, however it had a good performance when calculating force 
coefficients. The SIGMA model did not perform well in the low pressure gradient flow. After the analysis of the results yielded by the LES models in both geometries it can be determined that the VMS shows a better overall behavior than the other three models. This SGS model is used for the results in the following sections.

\subsection{Flow structures}

\subsubsection{Average flow structures}

According to Townsend's attached eddy hypothesis [27], the average large eddy motion consists of pairs of inviscid parallel roller vortices. These eddies extract energy from the mean flow through the vortex stretching mechanism. However, when the flow is bounded by a wall, the eddies are restricted and form double-cone eddies. Although Townsend's hypothesis holds for eddies spawning from a single point, these ideal flow structures have great resemblance to the structures observed in the wake of both the Ahmed and the Asmo cars.

Several authors have proposed methods for identifying coherent structures of the flow (see for instance Hunt et al. [28], Jeong and Fazle [29], Chong et al. [30]). In the present work the Q-criterion proposed by Hunt et al. [28] is chosen for the identification of the coherent structures. The method is based on the second invariant of the velocity gradient tensor, Q:

$$
Q=\frac{1}{2}\left(\left\|\Omega^{2}\right\|-\left\|S^{2}\right\|\right)
$$

where $\Omega$ is the rate-of-rotation tensor.

The method identifies a vortical structure as a spatial region where $Q>0$, i.e. where the rotation overcomes the strain. In figure 10, the time-averaged 
vortical structures for both the Ahmed and the Asmo cars are presented.

Figures 10a and 10b show Q-isocontours of the vortices generated in the wake of the cars. These structures were obtained from the mean flow. As can be seen, two longitudinal vortices are generated in the back of the cars. The presence of these vortices can also be verified by means of a local pressure minimum, shown in figures 10c and 10d.

These turbulent structures are found in both geometries. However, when comparing the vortices, the magnitude and strength of these structures in the Asmo car are lower, i.e. they are smaller in size, than those present in the Ahmed car. This fact is especially evident in figures 10c and 10d, where compared to the pressure isosurface of the Ahmed car, the vortex structure in the Asmo car is fragmented into smaller, different valued pressure isosurfaces making this eddy weaker in comparison to the one displayed by the Ahmed body flow. It can also be seen in figure 10 that the lower part of the vortices are bounded by the floor of the channel, which restricts the overall size of the eddy. ${ }^{1}$

Figure 11 shows the mean streamlines in the location where the main longitudinal vortices are generated. It is important to point out that even though the geometry in both cases is very different, i.e. the slant back in the Ahmed body and the curved profile of the Asmo car, the vortex generation mechanism seems to be the same. In both cases the following can be observed: i) a high speed stream moving along the side wall of the car; ii) a slow speed

\footnotetext{
${ }^{1}$ Figure 10 was obtained using a mesh containing more $\mathrm{CV}$ in the wake area. The total number of control volumes was $4.9 \times 10^{6}$ and $5.2 \times 10^{6}$ for the Ahmed and the Asmo cars, respectively. All other mesh parameters remained the same
} 
stream in the back of the car and, iii) the main flow traveling along the top of the body. In the Ahmed body, the slow speed stream consists of fluid recirculating along the slant wall, whereas in the Asmo car it is the main recirculation bubble in the back. The vortex is generated by the interaction between these three streams, as the high speed stream passes the end of the side wall it flows towards the mid plane (pushed by the low pressure zone found in the back), crashing into the slow speed stream, decelerating before colliding against the main flow closer to the mid plane (where the pressure has a higher value). This second collision changes the mixed stream direction giving it a twirling motion, creating the longitudinal vortex.

Figures $12 \mathrm{a}$ and $12 \mathrm{~b}$ show the recirculation bubbles behind the bodies. The recirculation non-dimensional length for the Ahmed body is around $0.69 h_{r e f}\left(0.65 h_{r e f}\right.$ in Krajnović and Davidson [2]), whereas for the Asmo body is around $0.48 h_{r e f}$. Besides from the size, the recirculation bubbles in both bodies differ in shape. In the Ahmed body it is composed of two straight span wise vortices generated by the flow coming down the slant back and the flow from the underbody. In the Asmo body, the vortex takes the shape of a horseshoe toroidal vortex bounded by the flow coming from the sides and the top of the body. The flow from the underbody, after passing through the diffuser, reduces its speed which decreases its influence in the recirculation bubble. This stream of fluid interacts with the toroidal vortex forming a smaller parallel vortex in the bottom of the recirculation bubble.

Also shown in figure 12a is the flow detachment and recirculation areas that are formed in the slant back in the Ahmed car. The sudden geometry change causes the fluid to separate from the surface. This separation comes 
with a pressure drop that eventually pulls the fluid back towards the surface. The space between the detachment and reattachment point converts into a recirculation area. Flow detachment is generally identified by a low pressure zone. The places where fluid separation occurs are accompanied by steep pressure gradients that generate vortices and eddies, which in turn augment drag on the body. Other factors affecting the aerodynamic drag, and which are partially responsible for the pressure drag a body experiences, are the high pressure bubbles in the front of the geometry and low pressure bubbles in the back.

Figures $13 \mathrm{a}$ and $13 \mathrm{~b}$ depict the non-dimensional pressure coefficient $\left(c_{p}\right)$ contours for the two bodies. These figures show that the pressure gradients in the Ahmed body are much greater than those of the Asmo body. Additionally, it can be seen that the Ahmed body has several zones of detachment. Furthermore, the pressure difference around the Ahmed car is larger than that of the Asmo car.

The mild pressure gradients in the Asmo body allow the flow to remain attached for most of the car length, producing a smaller wake as aforementioned. The steeper pressure gradients in the Ahmed body are strong enough to produce separated flow increasing the size of the recirculating bubble and pressure losses in the wake, and consequently increasing the pressure drag.

From figure 13, the high and low pressure bubbles around the bodies can be inferred. The low profile nose of the Asmo car allows the formation of a smoother flow pattern, diminishing the stagnation in the flow and reducing the size of the high pressure bubble. In the Ahmed car, the wider nose offers a larger resistance to the flow, increasing the size of the high pressure bubble. 
It is also important to notice that at the slant back, the edges are in a low pressure zone with high flow separation. This accounts for the formation of the big lifted longitudinal vortices shown before. There is also a mid-body low pressure zone present in both geometries. In the Ahmed car it is found near the front of the car, immediately after the nose, whereas this zone is spread wider in the Asmo geometry, accounting for the smoother pressure gradients of this geometry.

The effects of pressure gradients can be classified in two mayor groups, positive and negative relative to the direction of the flow. These zones can readily be seen in figure 13 .

\subsubsection{Instantaneous flow structures}

In this section, instantaneous coherent structures captured with two meshes are shown for comparison, for both the Ahmed and the Asmo cars. The main objective here is to assess the capabilities of the LES, using relative coarse grids, of capturing the large-scale flow structures. As it is shown hereafter, flow structures are well represented with the coarser grids for both car-like geometries. For the Ahmed car, the mesh of $1.6 \times 10^{6} \mathrm{CV}$ assessed in Sections 4.1 and 4.2 and, a finer grid of $4.9 \times 10^{6} \mathrm{CV}$ refined in the near wake zone (all other mesh parameters remained the same as in the $1.6 \times 10^{6} \mathrm{CV}$ mesh) were used. In a similar manner, for the Asmo car the mesh of $2.8 \times 10^{6} \mathrm{CV}$ and a wake refined one of $5.2 \times 10^{6} \mathrm{CV}$ were used.

Figures 14 to 17 show the $\mathrm{Q}$ isosurfaces in both the front and the rear of the cars. In figure 14, the structures formed in the front of the Ahmed car are shown. In this zone, the flow structure is smooth and resembles a laminarized flow. This part of the geometry has a negative pressure gradient 
(pressure decreases in the direction of the flow). This gradient "pushes" the fluid back, increasing the velocity gradient near the wall, thus increasing the local wall shear stress.

As the fluid moves back, it reaches the local pressure minimum and thus, a zone of positive pressure gradient. In figure 14, this point is shown as the transition of the smooth coherent structure to the unstable flow along the top of the car. This zone is characterized by a separation region and a lower wall shear. This is depicted for both meshes in the figure as a collection of small vortical structures lifting away from the wall. Several authors, such as Minguez et al. [3] and Krajnović and Davidson [12] stated there is a recirculation zone here. Even though the meshes used in the present study are not fine enough for a complete resolution of the flow in this area, evidence (pressure field and coherent structures) suggests the presence of recirculation in this place. Further back, the pressure gradient becomes small and the coherent structures in the Ahmed car exhibit the turbulent behavior to be expected from the streak instability cycle. A close inspection of figure 14 shows a small structure, generated in the lower corner of the car. As was exposed by Krajnović and Davidson [2], this vortical structure is generated by the interaction of the boundary layers of the floor and the underbody of the Ahmed car.

The slant back is a geometry feature unique to the Ahmed car and it displays very turbulent flow. Figure 15 shows the Q isosurfaces in this region. Both meshes are capable of well capturing the small vortical structures forming on the surface, right at the edge where the slanted surface starts, as well as, several big vortical structures. These structures are the vortices 
that form the recirculation bubble, the big lifted stream-wise vortices (shown earlier) and large horseshoe-like vortices shed from the slant wall.

The small vortical structures are the result of the change in surface direction and the subsequent negative pressure gradient generated. As was explained earlier, this pressure gradient creates flow instabilities, flow detachment, recirculation and flow reattachment. The combination of the flow instabilities and the geometry present add up to vortex shedding in this section.

The flow structures in front of the Asmo car are different than those observed in the Ahmed car (see figure 16). After passing the stagnation point (zone with no structure visible), the mild negative pressure gradient along the nose of the car permits the flow to be more stable than that found in the Ahmed car. Here, the flow over this geometry show very little instabilities. However, evidence of the behavior explained earlier can be found. Even with the coarser grid (see figure 16a), the flow structures formed can readily be observed. As the fluid travels through the top of the nose it reaches a local pressure minimum. In this region, the pressure gradient becomes larger and a smoother structure that lightly resembles that of the Ahmed car forms. Upon reaching the low pressure point, the structure breaks down due to the negative pressure gradient. Furthermore, the small vortical structures in the bottom of the car, are vortices shed around the tires.

The turbulent structures in the back of the Asmo car are different in shape and size as those seen in the Ahmed car. Figure 17 shows smaller vortical structures being generated in the rear of the geometry. These structures are well represented even by the coarse grid. The vortex shedding phenomenon 
can be witnessed readily in this geometry; coherent structures detach from the bottom of the car, as well as small vortices detaching from the wheels, the diffuser and the trailing edge of the body. Two large structures, corresponding with the big stream-wise vortices aforementioned are also seen. As it is expected in flow over buff bodies, both geometries show vortex shedding. However, in the Ahmed car, especially in the slant back, this phenomenon is very strong.

\section{Conclusions}

Challenging large-eddy simulations were carried out in two different geometries, the Asmo and the Ahmed cars, using four different turbulence models, the QR, the VMS, the SIGMA and the WALE. To the authors knowledge, this is the first time the SIGMA, QR and VMS models are used in resolving flow around simplified car models.

When comparing the results obtained in the Ahmed geometry with the experimental results it can be seen that the agreement in the slanted back is acceptable. Considering the coarse meshes used, results in the near wake show fairly good results when compared to the experimental data. The results of the Asmo car are also in quite good agreement with experimental results. Though they are not as accurate as results from the literature with

finer meshes, comparisons here presented using such coarse grids are useful to evaluate the potential of coarse LES simulations.

Even though the grids used are quite coarse, coherent structures have been well captured. These structures in the flow around the Asmo car are much weaker and exhibit less turbulent behavior than those found around 
the Ahmed car. The largest structures in the flow, the lifted stream-wise longitudinal vortices in the wake, are present in both geometries using the same generation mechanism. However, the geometry features of the Ahmed body allow for the formation of a much stronger vortex.

The recirculation bubble present in both geometries differ in both shape and size. The one present in the Asmo car is smaller, shows a horseshoe shape and is formed by flow coming from the top and the sides of the car. The bubble found in the Ahmed car is formed by two span-wise vortices and is created by the flow coming from the top and the bottom of the geometry. The differences in this structure allow for different magnitudes in the pressure fields, being the pressure behind the Ahmed car lower than that found in the Asmo car.

Another difference in pressure is also found in the nose of the car. The low profile nose of the Asmo car results in less stagnation and lower pressures in the front. The Asmo car also exhibits lower pressure gradients around the entire body. This produces a more laminarized flow around the entire geometry, less vortical structures detaching and less vortex shedding in its back.

All the differences in the flow features explained in the previous paragraphs account for the results shown in tables 3, 4, 5 and 6; the drag coefficient for the Ahmed car is higher than that calculated around the Asmo car. Additionally, the Ahmed car shows a large positive lift coefficient, opposed to the smaller value found for the Asmo car.

It should be pointed out that the LES turbulence models perform quite well in coarse meshes thanks to the conservative formulation used. The 
SIGMA and VMS models performed very similarly and better than the WALE and QR models in the Ahmed car, where the geometry changes and corners heavily influence the flow. QR and VMS models performed better than WALE and SIGMA models in the Asmo car. However, the VMS model showed better behavior as it deals quite well with flows heavily influenced by the boundary layer and by geometrical features. Overall analysis of the models leads to the conclusion that, from a practical viewpoint, the recommended model to solve cases involving flow past car-like bodies is the VMS model.

\section{Acknowledgments}

This work has been partially financially supported by the "Ministerio de Economía y Competitividad, Secretaría de Estado de Investigación, Desarrollo e Innovación", Spain (Ref. ENE2010-17801), by the collaboration project between "Universitat Politècnica de Catalunya - BarcelonaTech" and Termo Fluids S.L. and, the "Departamento Administrativo de Ciencia, Tecnología e Innovación - Colciencias" through their doctoral training program "Francisco Jose de Caldas". Special thanks to Sinisa Krajnović, Eric Serre and Matthieu Minguez for providing results to permit comparison in the Ahmed car.

\section{References}

[1] S. R. Ahmed, G. Ramm, G. Faltin, Some Salient features of the time averaged ground vehicle wake, journal $=$ SAE paper no 840300, booktitle 
$=$, publisher $=$, project $=$, institution $=$, school $=$, year $=1984$, volume $=$, number $=$, pages $=$.

[2] S. Krajnović, L. Davidson, Flow around a simplified car: Part1: Large eddy simulation, journal = ASME: Journal of Fluids Engineering, booktitle $=$, publisher $=$, project $=$, institution $=$, school $=$, year $=2005$, volume $=127$, number $=$, pages $=907-919$.

[3] M. Minguez, R. Pasquetti, E. Serre, High Order Large Eddy Simulation of Flow Over the "Ahmed Body" Car Model., Physics of Fluids 20.

[4] M. Serre, E. Minguez, R. Pasquetti, E. Guilmineau, G. Deng, M. Kornhaas, M. Schäfer, J. Fröhlich, C. Hinterberger, R. W., On simulating the turbulent flow around the Ahmed body: A French-German collaborative evaluation of LES and DES, Computers \& Fluids .

[5] O. Lehmkuhl, R. Borrell, C. D. Pérez-Segarra, A. Oliva, R. Verstappen, LES modeling of the turbulent flow over an Ahmed car, in: ERCOFTAC WORKSHOP Direct and Large-Eddy Simulations 8, 89-94, 2010.

[6] S. Perzon, L. Davidson, On Transient Modeling of the Flow Around Vehicles Using the Reynolds Equation., in: ACFD 2000 Beijing, China, $720-727,2000$.

[7] T. Nakashima, M. Tsubokura, T. Nouzawa, T. Nakamura, H. Zhang, N. Oshima, Large-Eddy Simulation of Unsteady Vehicle Aerodynamics and Flow Structures, in: BBAA VI International Colloquium on: Bluff Bodies Aerodynamics \& Applications, 2008. 
[8] H. Lienhart, C. Stoots, S. Becker, Flow and Turbulence Structures in the Wake of a Simplified Car Model (Ahmed Model), in: DGLR Fach. Symp. der AG STAB, Stuttgart University., 2000.

[9] S. Jakirlić, R. Jester-Zücker, C. Tropea, 9th ERCOFTAC/IAHR/COST Workshop on Refined Turbulence Modelling, 2001.

[10] C. Hinterberger, M. García-Villalba, W. Rodi, Large Eddy Simulation of Flow Around the Ahmed Body. The Aerodynamics of Heavy Vehicles: Trucks, Buses, and Trains, Numerical Heat Transfer, Part B (2004) 7788.

[11] S. Kapadia, S. Roy, M. Vallero, K. Wurtzler, J. Forsythe, DetachedEddy Simulation Over a Reference Ahmed Car Model, in: Direct and Large Eddy Simulations V (ERCOFTAC series), 481-488, 2004.

[12] S. Krajnović, L. Davidson, Flow Around a Simplified Car: Part2: Understanding the Flow, ASME: Journal of Fluids Engineering 127 (2005) 919-928.

[13] D. Aronson, S. Brahim, S. Perzon, Multigrid heat transfer calculations using different iterative schemes, SAE Technical Paper 2000-01-0485 .

[14] M. Tsubokura, T. Kobayashi, T. Nakashima, T. Nouzawa, T. Nakamura, H. Zhang, K. Onishi, N. Oshima, Computer Visualization of Unsteady Flow Around Vehicles Using High Performance Computing, Computers and Fluids 38 (2009) 981 - 990.

[15] XFLOW, Aerodynamic Analysis Involving Moving Parts with Xflow, Tech. Rep., 2010. 
[16] F. Nicoud, F. Ducros, Subgrid-scale stress modeling based on the square of the velocity gradient tensor, Flow, Turbulence and Combustion 62 (1999) 183-200.

[17] T. J. R. Hughes, L. Mazzei, K. E. Hanzen, Large Eddy Simulation and the variational multiscale method., Computing and Visualization in Science 3 (1) (2000) 47-59.

[18] F. Nicoud, H. Toda, O. Cabrit, S. Bose, , J. Lee, Using Singular Values to Build a Subgrid-Scale Model for Large Eddy Simulations, Physics of fluids 23.

[20] T. Hughes, G. Feijoo, L. Mazzei, J. Quincy, The variational multiscale method - a paradigm for computational mechanics, Computer methods in applied mechanics and engineering 166 (1998) 3-24.

[21] A. W. Vreman, The Filtering Analog of the Variational Multiscale Method in Large-Eddy Simulation, Physics of Fluids 15 (8).

[23] R. W. C. P. Verstappen, A. E. P. Veldman, Symmetry-preserving Discretization of Turbulent Flow, Journal of Computational Physics 187 (1) (2003) 343-368.

[25] O. Lehmkuhl, C. D. Pérez-Segarra, R. Borrell, M. Soria, A. Oliva, TERMOFLUIDS: A new Parallel unstructured CFD code for the simulation of turbulent industrial problems on low cost PC Cluster, in: Proceedings of the Parallel CFD 2007 Conference, 1-8, 2007. 
[26] G. Karypis, V. Kumar, Multilevel k-way Partitioning Scheme for Irregular Graphs, Journal Of Parallel And Distributed Computing 48 (1998) 96-129.

[27] A. A. Townsend, The Structure of Turbulent Shear Fow, Cambridge University Press, 1976.

[28] J. C. R. Hunt, A. A. Wray, P. Moin, Eddies, streams, and convergence zones in turbulent flows, Tech. Rep., Center for Turbulence Research, 1988.

[29] J. Jeong, F. Hussain, On the Identification of a Vortex, Journal of Fluid Mechanics 285 (1995) 69-94.

[30] M. S. Chong, A. E. Perry, B. J. Cantwell, A General Classification of Three-Dimensional Flow Fields, Physics of Fluids 2 (5) (1990) 765-777.

[31] M. Germano, Differential filters for the large eddy numerical simulation of turbulent flows, Physics of Fluids 29 (1986) 1755-1757.

[32] A. W. Vreman, The adjoint filter operator in large-eddy simulation of turbulent flow, Physics of Fluids 16 (6) (2004) 2012, doi: 10.1063/1.1710479.

[33] P. Sagaut, M. Ciardi, A finite-volume variational multiscale method coupled with a discrete interpolation filter for large-eddy simulation of isotropic turbulence and fully developed channel flow, Physics of Fluids 18 (11) (2006) 115101, doi:10.1063/1.2391133. 


\section{References}

[1] S. R. Ahmed, G. Ramm, G. Faltin, Some salient features of the time averaged ground vehicle wake, SAE paper no 840300 .

[2] S. Krajnović, L. Davidson, Flow around a simplified car: Part1: Large eddy simulation, ASME: Journal of Fluids Engineering 127 (2005) 907919.

[3] M. Minguez, R. Pasquetti, E. Serre, High-order large-eddy simulation of flow over the Ahmed body car model, Physics of Fluids 20 (9) (2008) 095101, doi:10.1063/1.2952595.

[4] M. Serre, E. Minguez, R. Pasquetti, E. Guilmineau, G. Deng, M. Kornhaas, M. Schäfer, J. Fröhlich, C. Hinterberger, R. W., On simulating the turbulent flow around the Ahmed body: A French-German collaborative evaluation of LES and DES, Computers \& Fluids .

[5] O. Lehmkuhl, R. Borrell, C. D. Pérez-Segarra, A. Oliva, R. Verstappen, LES modeling of the turbulent flow over an Ahmed car, in: ERCOFTAC WORKSHOP Direct and Large-Eddy Simulations 8, 89-94, 2010.

[6] S. Perzon, L. Davidson, On transient modeling of the flow around vehicles using the Reynolds equation, in: ACFD 2000 Beijing, China, 720-727, 2000.

[7] T. Nakashima, M. Tsubokura, T. Nouzawa, T. Nakamura, H. Zhang, N. Oshima, Large-eddy simulation of unsteady vehicle aerodynamics and flow structures, in: BBAA VI International Colloquium on: Bluff Bodies Aerodynamics \& Applications, 2008. 
[8] H. Lienhart, C. Stoots, S. Becker, Flow and turbulence structures in the wake of a simplified car model (Ahmed model), in: DGLR Fach. Symp. der AG STAB, Stuttgart University., 2000.

[9] S. Jakirlić, R. Jester-Zücker, C. Tropea, 9th ERCOFTAC/IAHR/COST Workshop on Refined Turbulence Modelling, 2001.

[10] C. Hinterberger, M. García-Villalba, W. Rodi, Large eddy simulation of flow around the Ahmed body. The aerodynamics of heavy vehicles: trucks, buses, and trains, Numerical Heat Transfer, Part B (2004) 77-88.

[11] S. Kapadia, S. Roy, M. Vallero, K. Wurtzler, J. Forsythe, Detached-eddy simulation over a reference Ahmed car model, in: Direct and Large Eddy Simulations V (ERCOFTAC series), 481-488, 2004.

[12] S. Krajnović, L. Davidson, Flow around a simplified car: Part2: Understanding the flow, ASME: Journal of Fluids Engineering 127 (2005) 919-928.

[13] D. Aronson, S. Brahim, S. Perzon, Multigrid heat transfer calculations using different iterative schemes, SAE Technical Paper 2000-01-0485 .

[14] M. Tsubokura, T. Kobayashi, T. Nakashima, T. Nouzawa, T. Nakamura, H. Zhang, K. Onishi, N. Oshima, Computer visualization of unsteady flow around vehicles using high performance computing, Computers and Fluids 38 (2009) 981 - 990.

[15] XFLOW, Aerodynamic analysis involving moving parts with Xflow, Tech. Rep., 2010. 
[16] F. Nicoud, F. Ducros, Subgrid-scale stress modelling based on the square of the velocity gradient tensor, Flow, Turbulence and Combustion 62 (1999) 183-200, doi:10.1023/A:1009995426001.

[17] T. Hughes, L. Mazzei, K. Jansen, Large Eddy Simulation and the variational multiscale method, Computing and Visualization in Science 3 (2000) 47-59, doi:10.1007/s007910050051.

[18] F. Nicoud, H. Toda, O. Cabrit, S. Bose, J. Lee, Using singular values to build a subgrid-scale model for large eddy simulations, Physics of Fluids 23 (2011) 085106, doi:10.1063/1.3623274.

[19] R. Verstappen, When Does Eddy Viscosity Damp Subfilter Scales Sufficiently?, Journal of Scientific Computing 49 (1) (2011) 94-110, doi: 10.1007/s10915-011-9504-4.

[20] T. Hughes, G. Feijoo, L. Mazzei, J. Quincy, The variational multiscale method - a paradigm for computational mechanics, Computer methods in applied mechanics and engineering 166 (1998) 3-24.

[21] A. W. Vreman, The filtering analog of the variational multiscale method in large-eddy simulation, Physics of Fluids 15 (8) (2003) L61, doi: 10.1063/1.1595102.

[22] O. Lehmkuhl, I. Rodríguez, A. Baez, A. Oliva, C. Pérez-Segarra, On the Large-Eddy Simulations for the flow around aerodynamic profiles using unstructured grids, Computers\&Fluids 84 (2013) 176-189.

[23] V. R., R. Camussi, Numerical experimenrs on strongly turbulent thermal 
convection in a slender cylindrical cell, Journal of Fluid Mechanics 477 (2003) 19-49.

[24] F. Trias, O. Lehmkuhl, A self-adaptive strategy for the time integration of Navier-Stokes equations, Numerical Heat Transfer. Part B 60 (2) (2011) 116-134, doi:10.1080/10407790.2011.594398.

[25] O. Lehmkuhl, C. D. Perez-Segarra, R. Borrell, M. Soria, A. Oliva, TERMOFLUIDS: A new Parallel unstructured CFD code for the simulation of turbulent industrial problems on low cost PC Cluster, in: I. Tuncer, U. Gülcat, D. Emerson, K. Matsuno (Eds.), Proceedings of the 2005 Parallel Computational Fluid Dynamics Conference, vol. 67 of Series: Lecture Notes in Computational Science and Engineering, Springer, 275$282,2007$.

[26] G. Karypis, V. Kumar, Multilevel k-way partitioning scheme for irregular graphs, Journal Of Parallel And Distributed Computing 48 (1998) 96-129.

[27] A. A. Townsend, The structure of turbulent shear flow, Cambridge University Press, 1976.

[28] J. Hunt, A. Wray, P. Moin, Eddies, stream and convergence zones in turbulent flows, Tech. Rep. CTR-S88, Center for turbulent research, 1988.

[29] J. Jeong, H. Fazle, On the identification of a vortex, Journal of Fluids Mechanics 285 (69-94), doi:10.1017/S0022112095000462. 
[30] M. S. Chong, a. E. Perry, B. J. Cantwell, A general classification of three-dimensional flow fields, Physics of Fluids A: Fluid Dynamics 2 (5) (1990) 765, doi:10.1063/1.857730.

[31] M. Germano, Differential filters for the large eddy numerical simulation of turbulent flows, Physics of Fluids 29 (1986) 1755-1757.

[32] A. W. Vreman, The adjoint filter operator in large-eddy simulation of turbulent flow, Physics of Fluids 16 (6) (2004) 2012, doi: 10.1063/1.1710479.

[33] P. Sagaut, M. Ciardi, A finite-volume variational multiscale method coupled with a discrete interpolation filter for large-eddy simulation of isotropic turbulence and fully developed channel flow, Physics of Fluids 18 (11) (2006) 115101, doi:10.1063/1.2391133. 


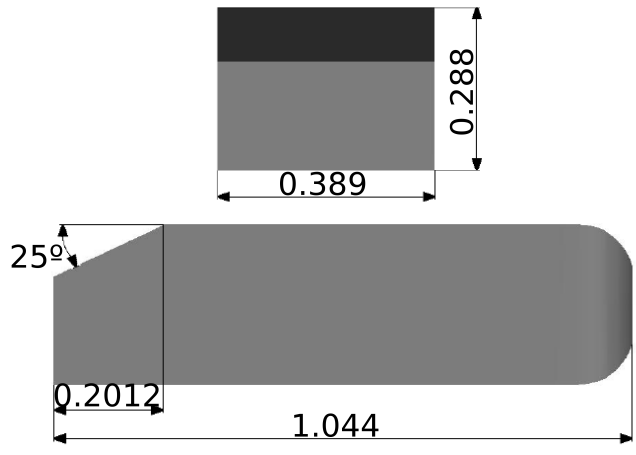

(a)

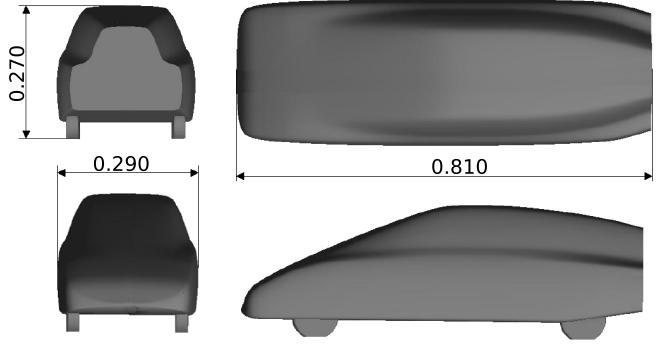

(b)

Figure 1: Model dimensions - Units in meters. (a) Ahmed car; (b) Asmo car

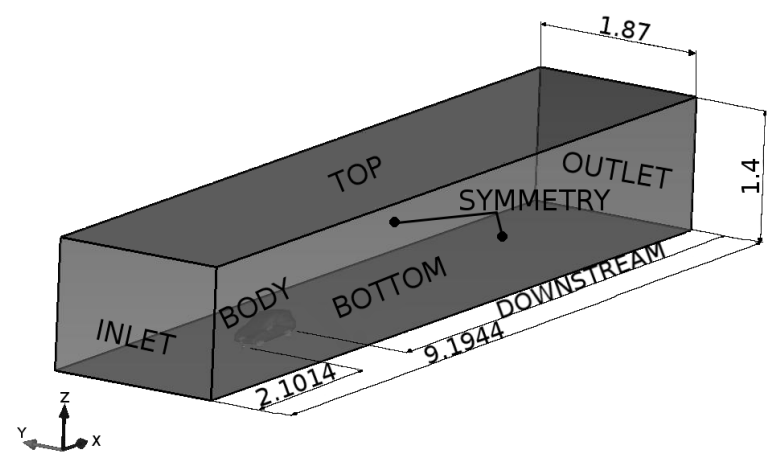

Figure 2: Computational domain - All dimensions in meters. 


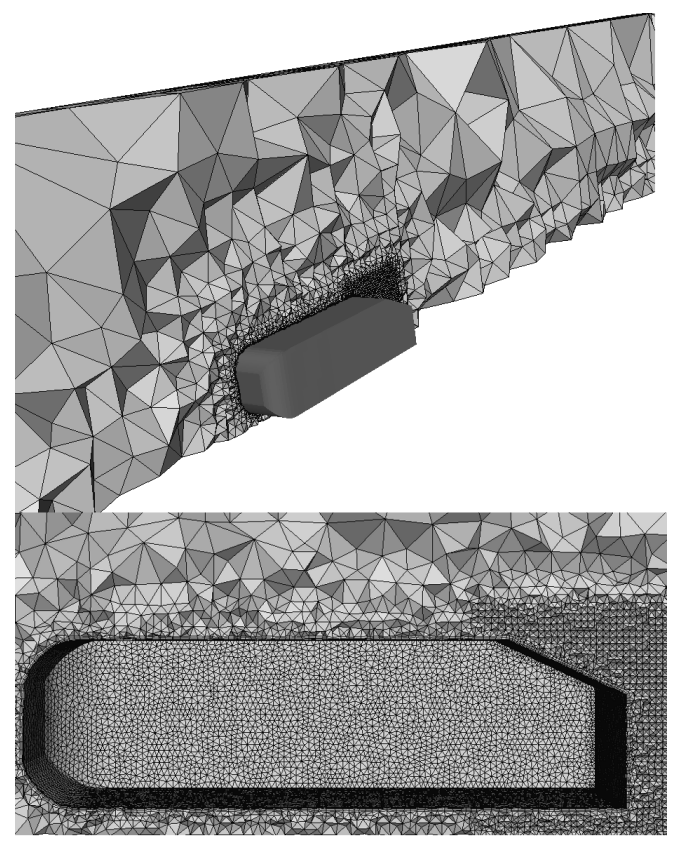

(a)

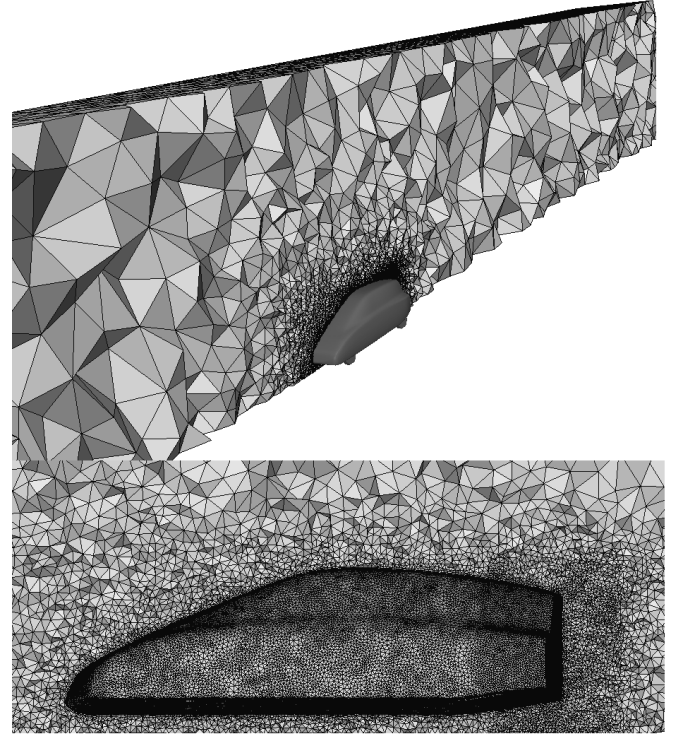

(b)

Figure 3: Visualization of the mesh in the channel. (a) Ahmed car; (b) Asmo car. 


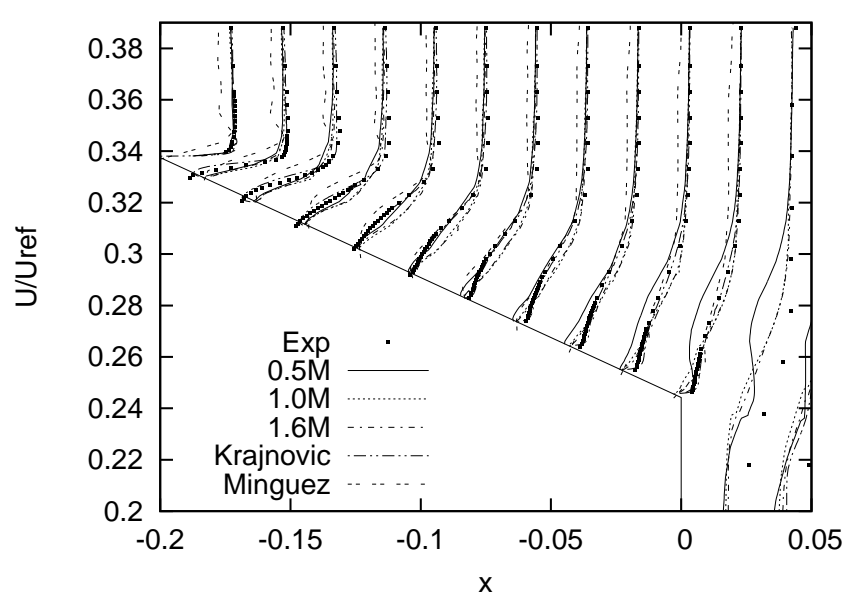

(a)

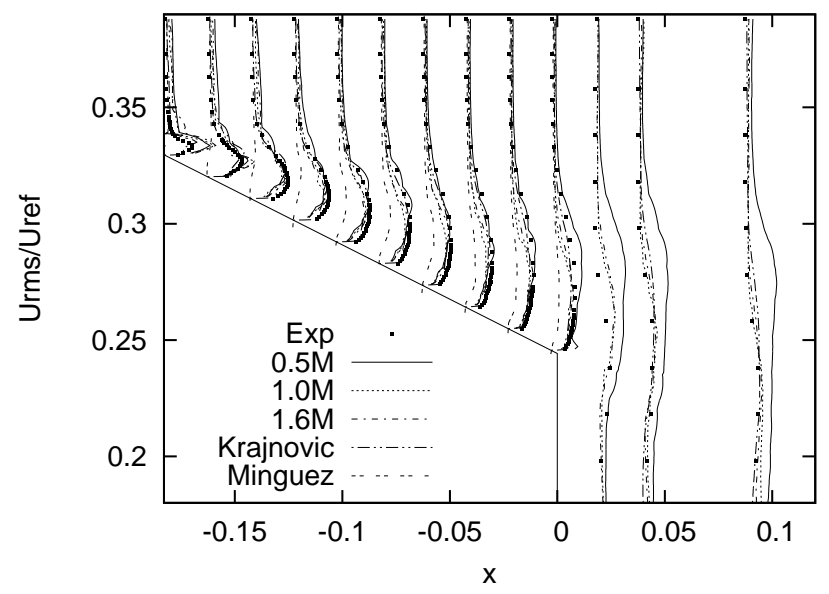

(b)

Figure 4: Mesh study for the Ahmed car. (a) Average stream-wise velocity and (b) its fluctuations, over the slant wall in the mid plane. 


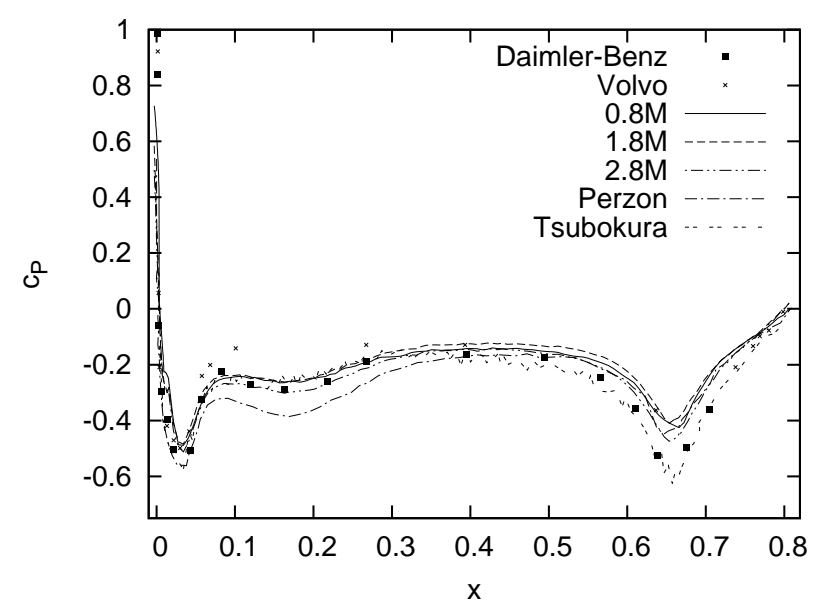

(a)

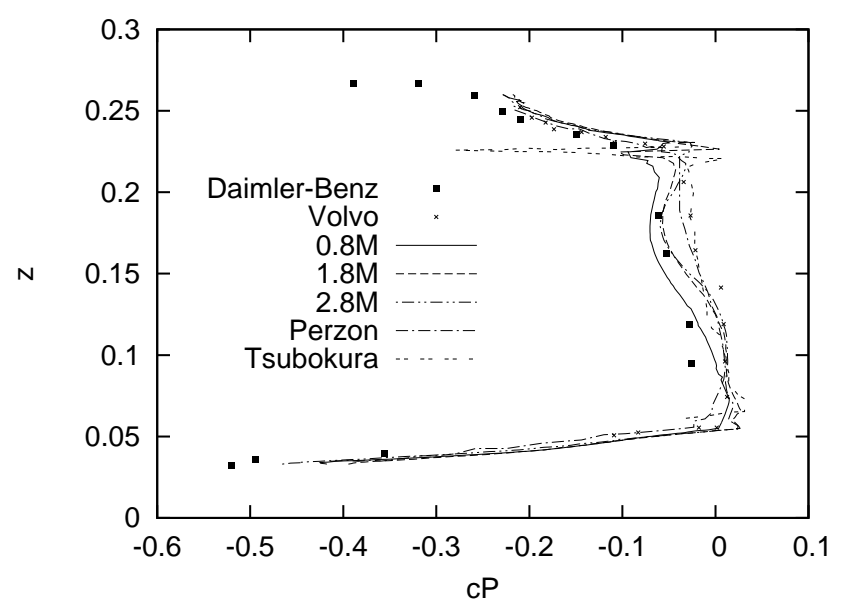

(b)

Figure 5: Mesh study for the Asmo car. (a) Average pressure coefficient in the underbody and (b) in the back. 

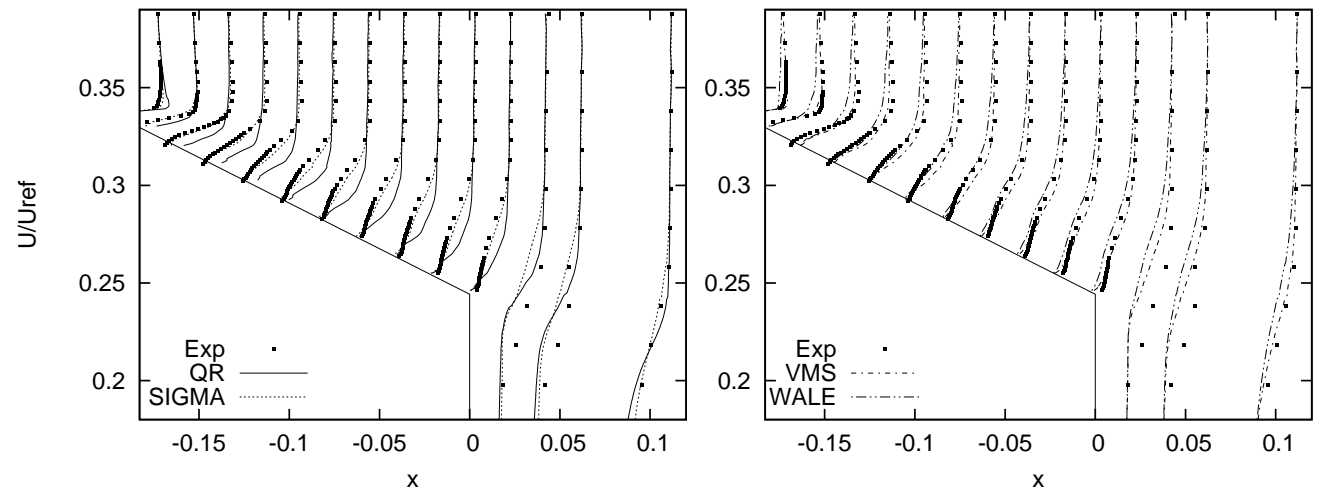

(a)
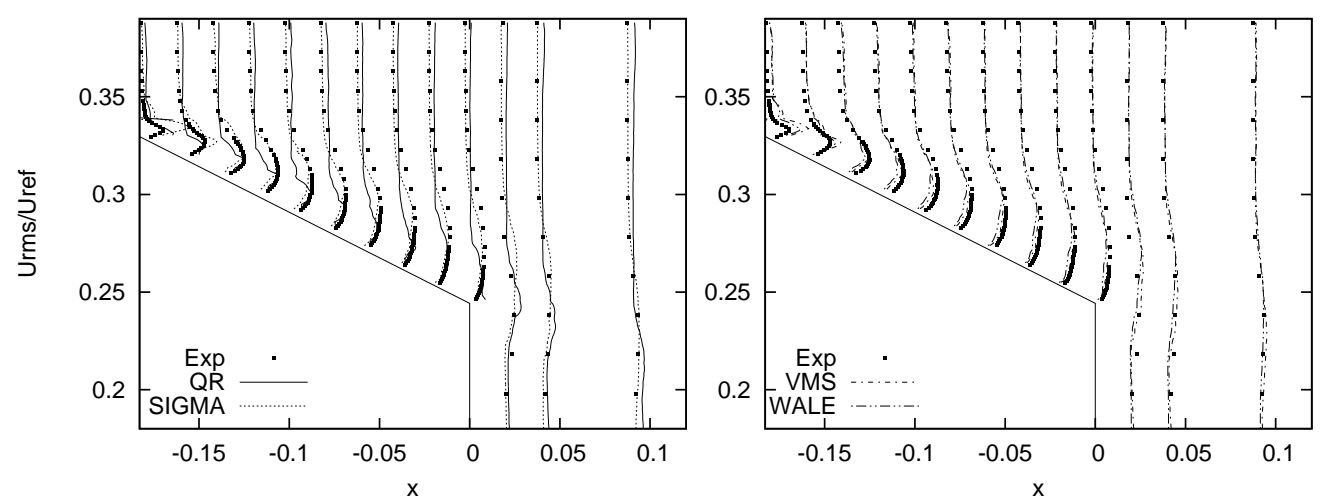

(b)

Figure 6: Models comparison. Velocity profiles over the slant wall and near wake in the mid plane for the Ahmed car. (a) Average stream-wise velocity. (b) Average stream-wise velocity fluctuations. 

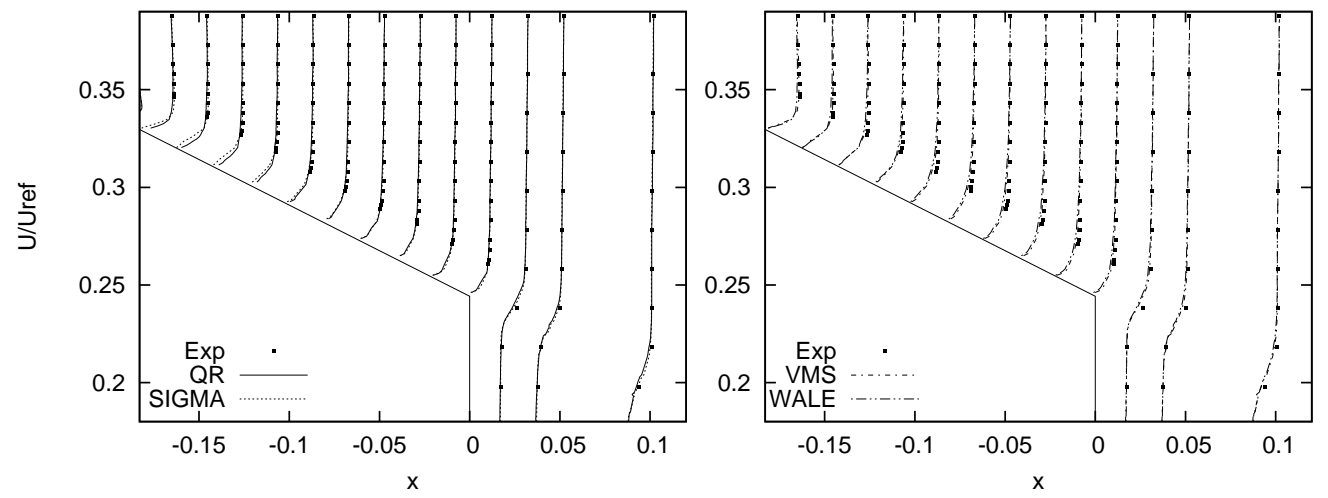

(a)
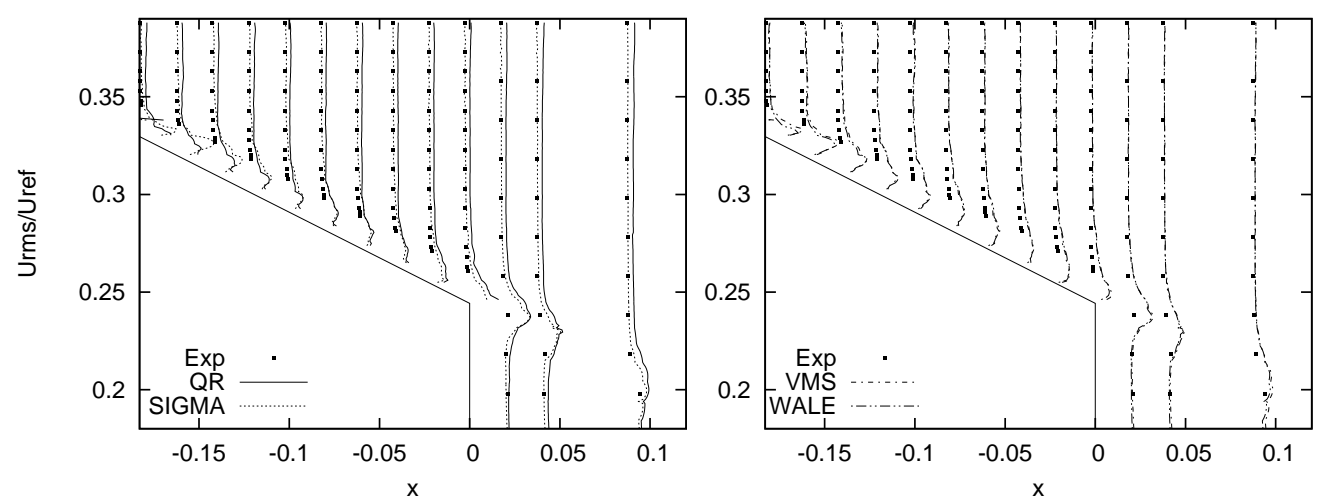

(b)

Figure 7: Models comparison. Velocity profiles over the slant wall and near wake in the $y=0.1$ plane for the Ahmed car. (a) Average stream-wise velocity. (b) Average streamwise velocity fluctuations. 


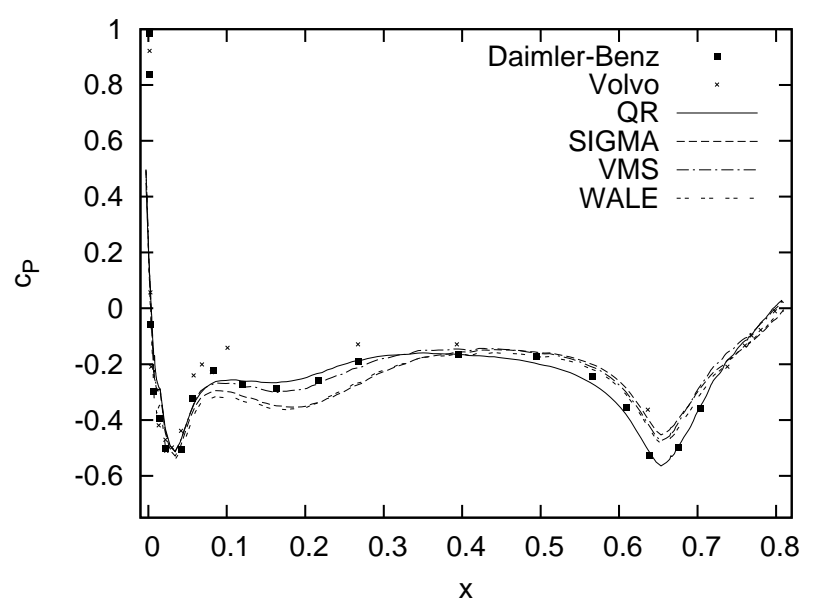

(a)

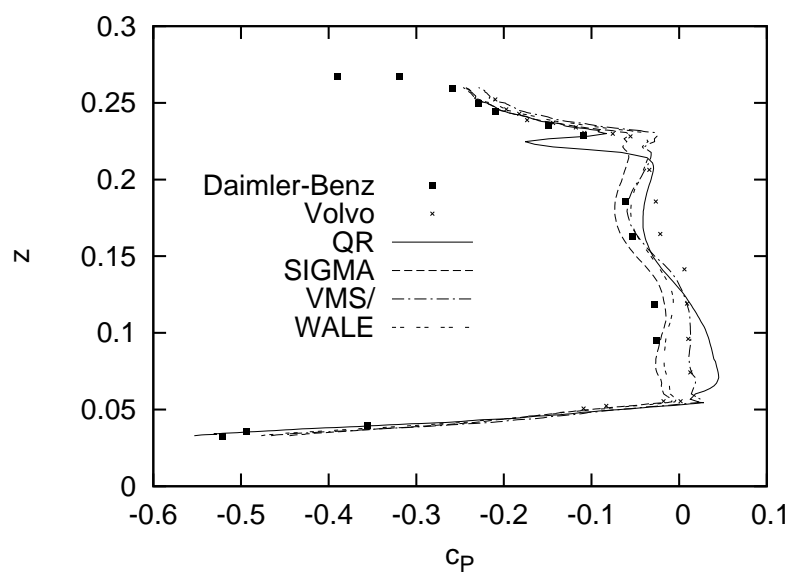

(b)

Figure 8: Pressure coefficients for the Asmo car. (a) In the underbody. (b) In the rear. 


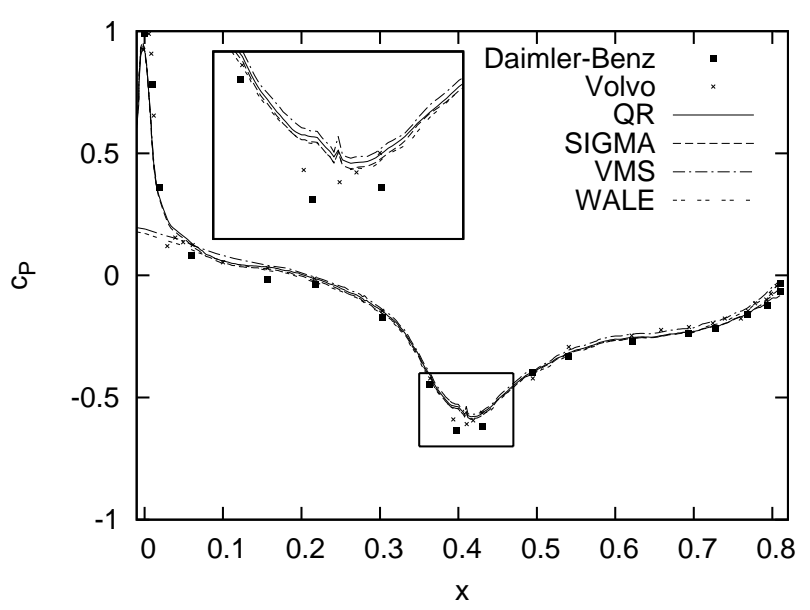

(a)

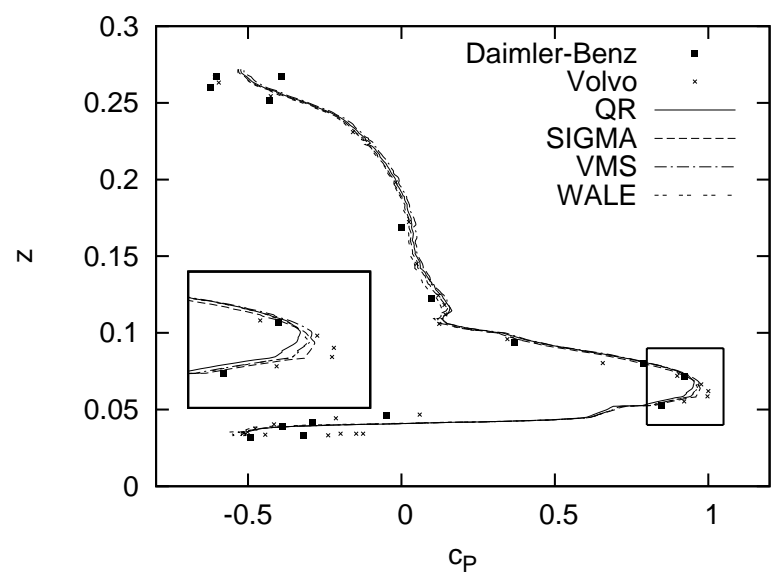

(b)

Figure 9: Pressure coefficients for the Asmo car. (a) In the roof. (b) In the frontal section. 


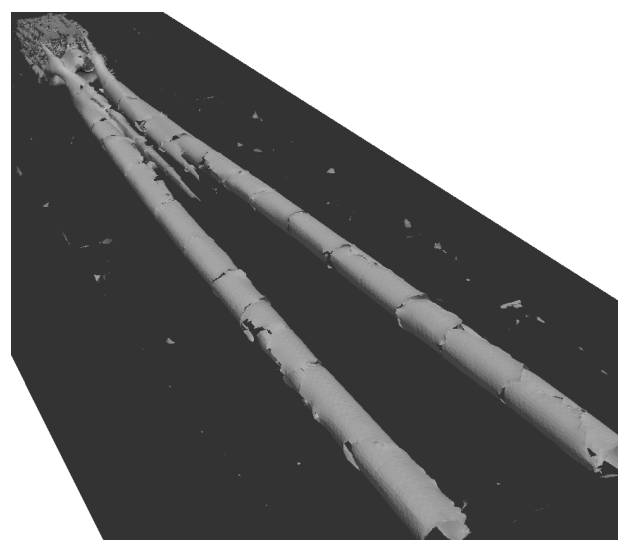

(a)

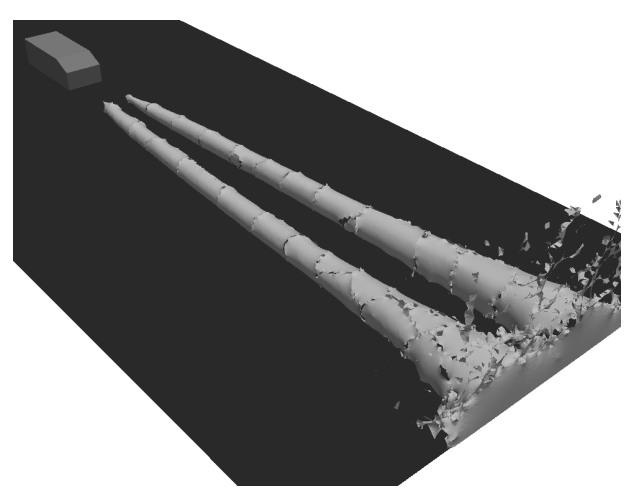

(c)

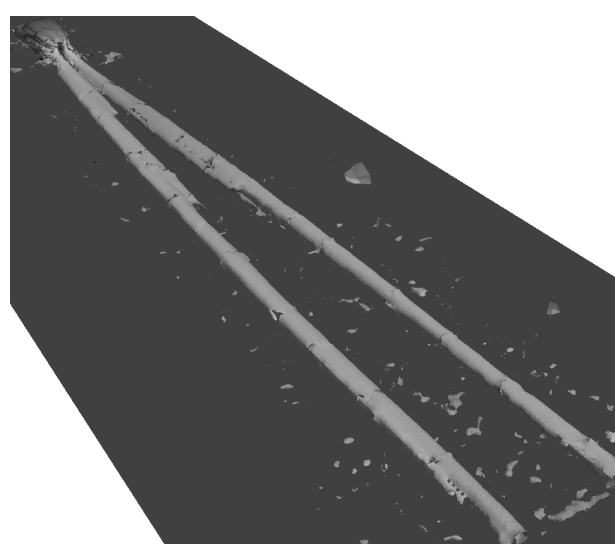

(b)

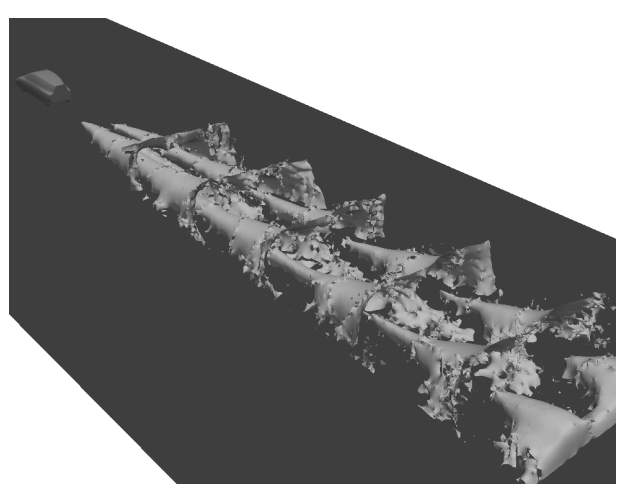

(d)

Figure 10: Time-averaged stream-wise vortical structures. (a) Ahmed car $Q=0.1$. (b) Asmo car $Q=0.1$. (c) Ahmed car $p=0$. (d) Asmo car $p=(-0.01,0.01)$ 


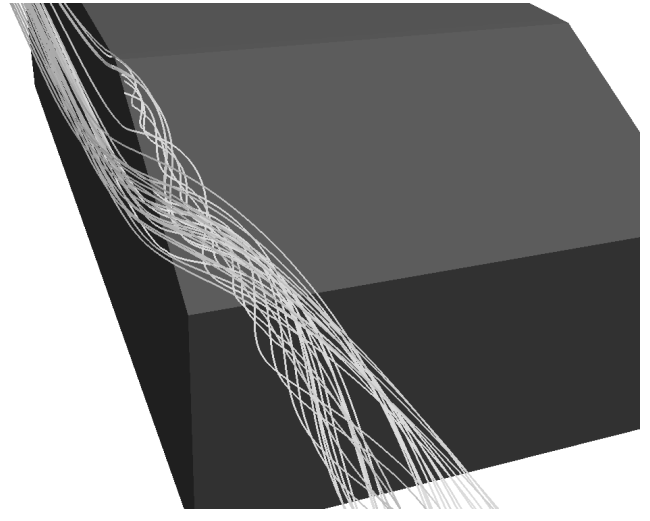

(a)

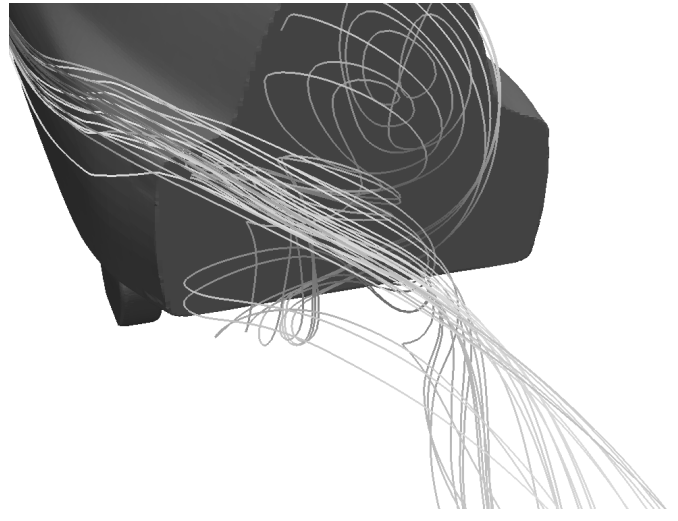

(b)

Figure 11: Time-averaged streamlines in the back of the car models. (a) Ahmed car. (b) Asmo car. 

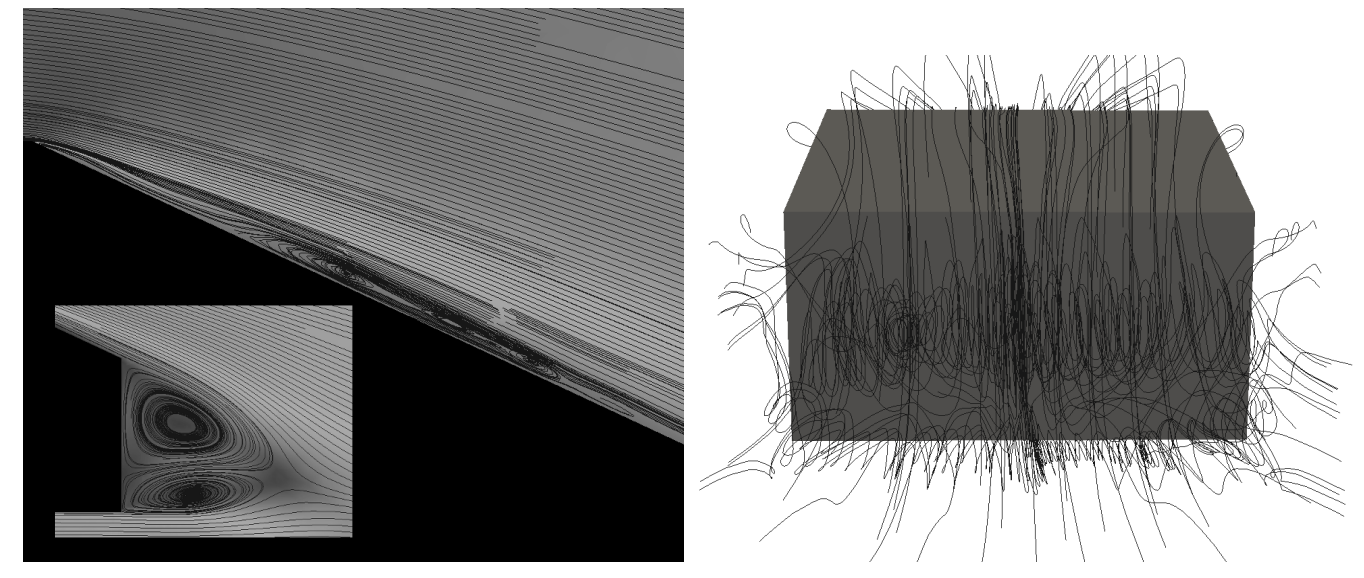

(a)
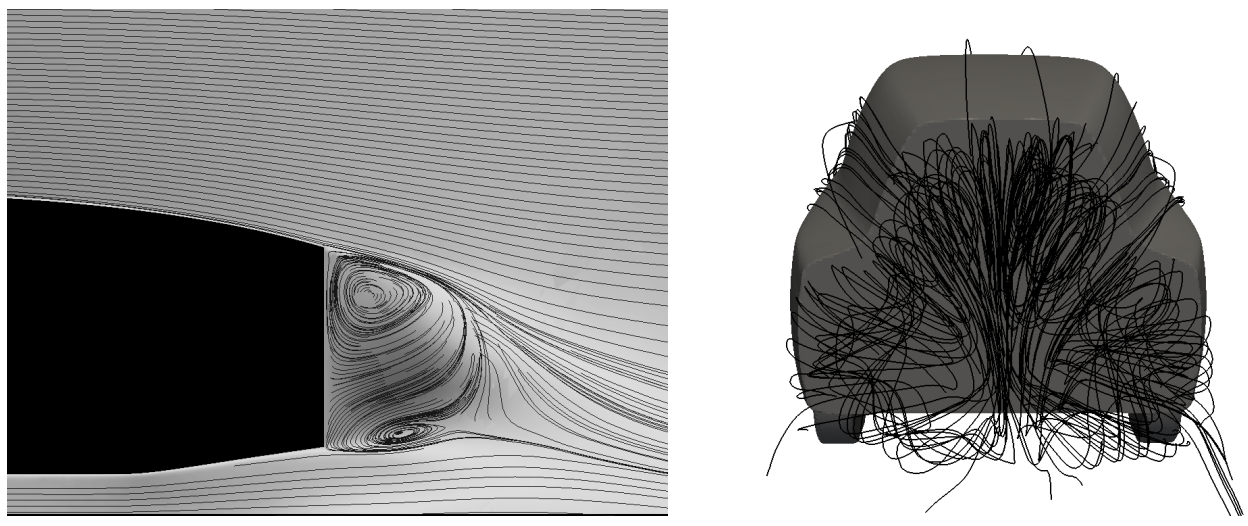

(b)

Figure 12: Recirculation bubble behind the geometries.(a) Ahmed car. (b) Asmo car. 

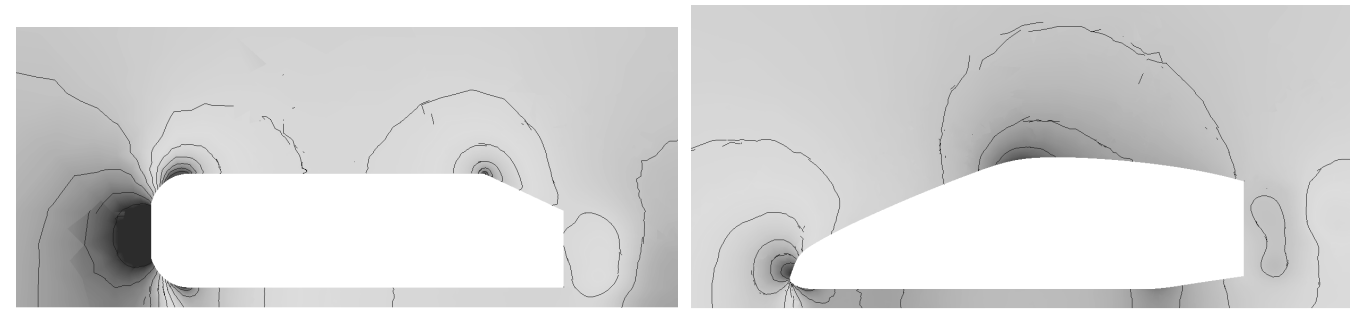

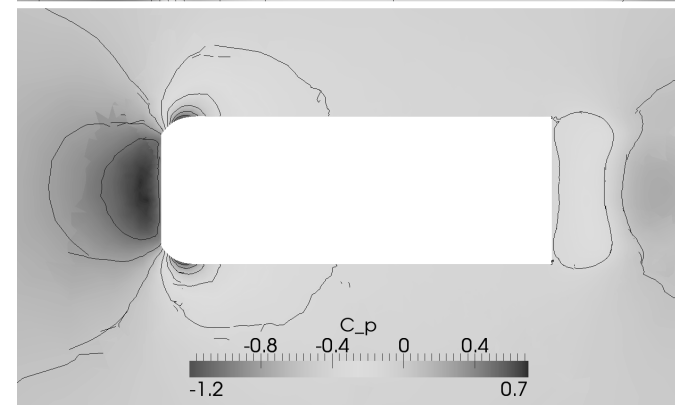

(a)

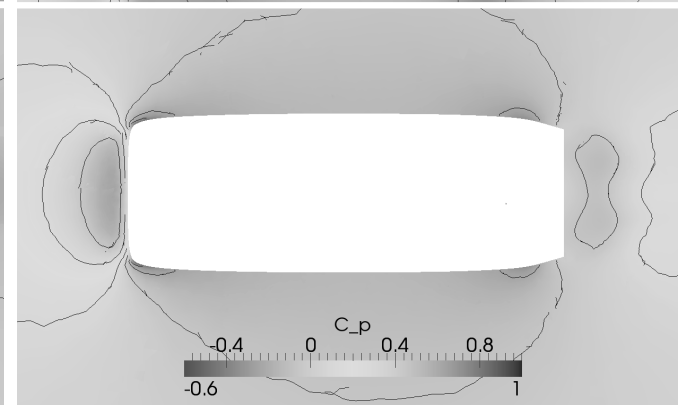

(b)

Figure 13: Time-averaged non-dimensional pressure contours.(a) Ahmed car. (b) Asmo car.

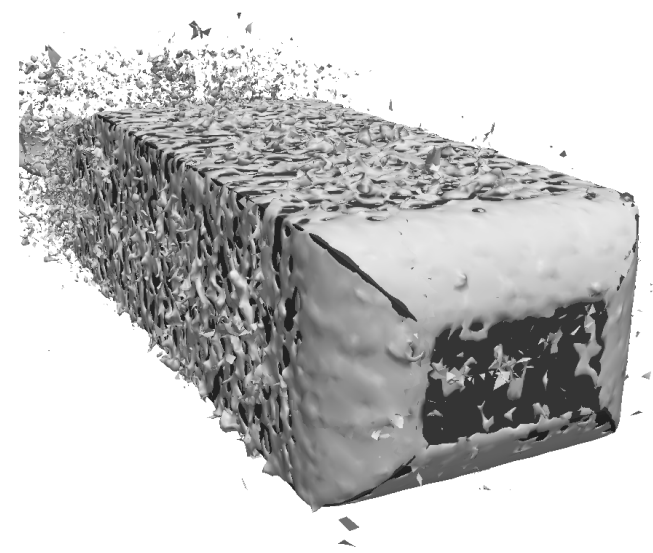

(a)

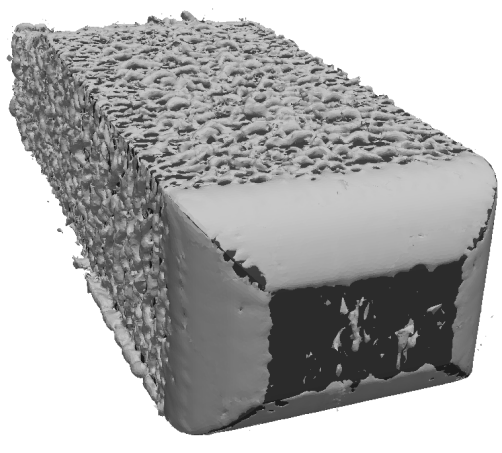

(b)

Figure 14: Instantaneous Q-isosurfaces in the front of the Ahmed car, $Q=20$. (a) $1.5 \times 10^{6}$ CV mesh. (b) $4 \times 10^{6} \mathrm{CV}$ mesh 


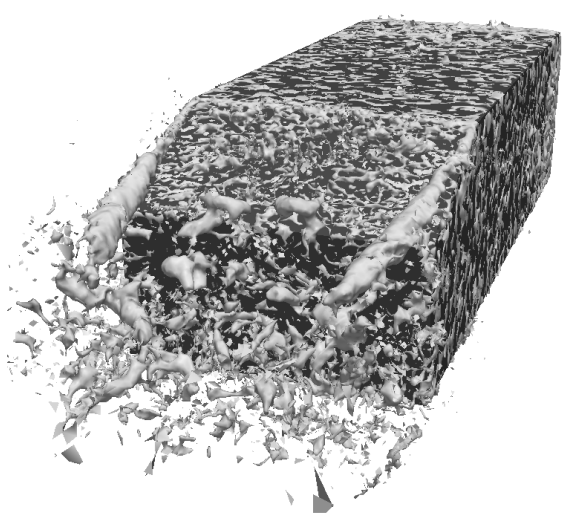

(a)

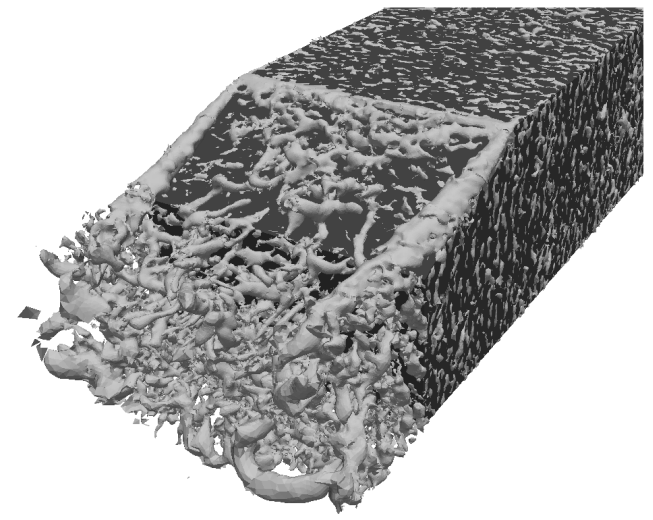

(b)

Figure 15: Instantaneous Q-isosurfaces in the rear of the Ahmed car, $Q=200$. (a) $1.5 \times 10^{6}$ CV mesh. (b) $4 \times 10^{6} \mathrm{CV}$ mesh

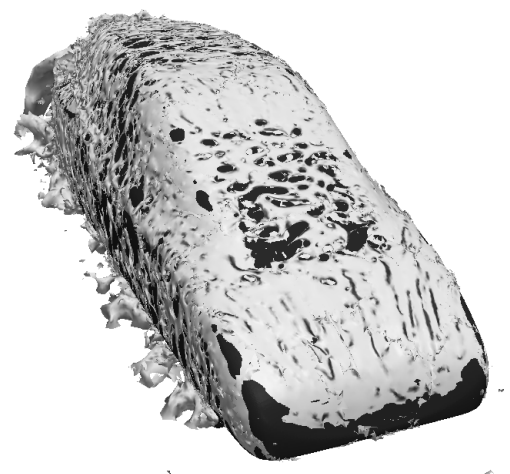

(a)

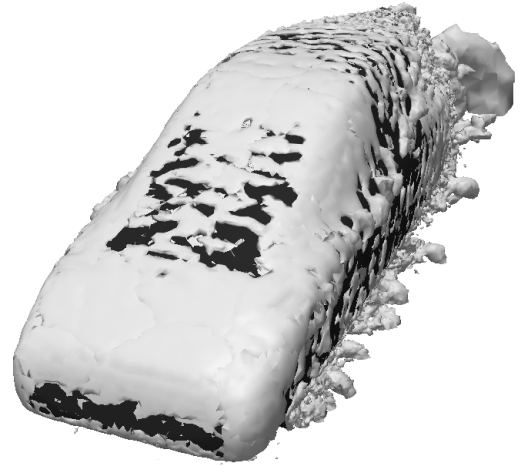

(b)

Figure 16: Instantaneous Q-isosurfaces in the front of the Asmo car, $Q=20$. (a) $2.8 \times 10^{6}$ CV mesh. (b) $5.3 \times 10^{6} \mathrm{CV}$ mesh. 


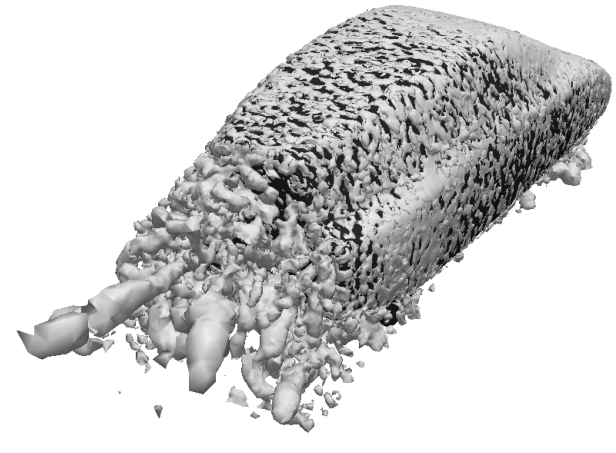

(a)

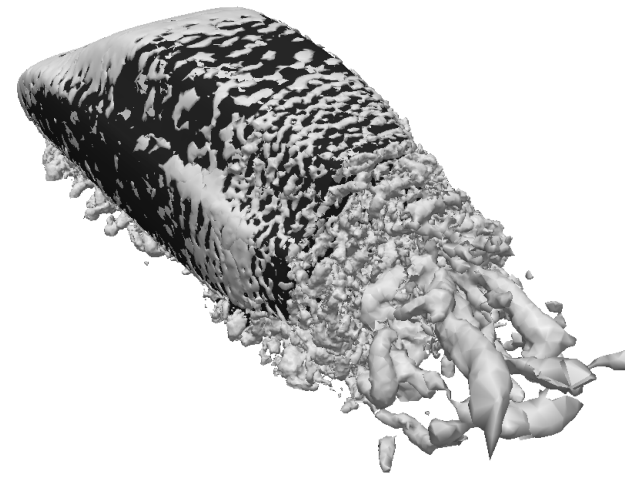

(b)

Figure 17: Instantaneous isosurfaces in the back of the Asmo car. $Q=200$. (a) $2.8 \times 10^{6}$ CV mesh. (b) $5.3 \times 10^{6} \mathrm{CV}$ mesh. 


\section{Appendix A. The VMS for finite-volume discretizations}

In the context of LES, scales are divided into the filtered quantity $(\bar{f})$ and the unresolved scales $\left(f^{u}\right)$. Thus, a generic quantity $f$ can be represented as:

$$
f=\bar{f}+f^{u}
$$

The VMS approach $[20,17]$ further separates the filtered quantity into large- and small-scales. If a second filter of length $\hat{l}$ (filter test) is applied to the filtered quantity, then both large- $(\hat{f})$ and small-scales $\left(f^{\prime}\right)$ are obtained.

$$
\bar{f}=\hat{f}+f^{\prime}
$$

The VMS introduced by Hughes et al. [20, 17] as a framework for SGS modeling for a Galerkin space-time formulation, was later extended to the physical space by Vreman [21]. As shown by Vreman [21], the equations for the large- and small-scales in the Fourier space when the filter is a projection in a Galerkin method are identical to those in the physical space.

The separation of scales in this work is carried out by using a non-uniform Laplace filter based on a Gaussian filter [31]. The second-order term in the Taylor expansion of $\mathcal{F} \bar{f}$ for a Gaussian convolution filter is the Laplace operator [32].

$$
\mathcal{F} \bar{f}=\left(\mathbf{I}+\frac{\hat{l}^{2}}{24} \nabla^{2}\right) \bar{f}
$$

where $\mathcal{F}$ is the filter transfer function. This filter is normalized, conservative and self-adjoint, which are desirable characteristics in order to preserve 
both symmetry and conservation properties of the continuous differential operators. It is also worth noting that the filter can be discretized in an identical manner than the diffusive term in equation 1.

With this separation of scales, it is possible to write the equations for the large- (eq. A.4) and small-scales (eq. A.5) as in [21], which reads,

$$
\begin{gathered}
\frac{\partial \hat{\overline{\mathbf{u}}}}{\partial t}+(\hat{\overline{\mathbf{u}}} \cdot \nabla) \hat{\overline{\mathbf{u}}}-\nu \nabla^{2} \hat{\mathbf{u}}+\rho^{-1} \nabla \hat{\bar{p}}=-\nabla \cdot \hat{\tau} \\
\frac{\partial \overline{\overline{\mathbf{u}}^{\prime}}}{\partial t}+\left(\overline{\mathbf{u}^{\prime}} \cdot \nabla\right) \overline{\mathbf{u}^{\prime}}-\nu \nabla^{2} \overline{\mathbf{u}^{\prime}}+\rho^{-1} \nabla \overline{p^{\prime}}=-\nabla \cdot \tau^{\prime}
\end{gathered}
$$

Adding up equations A.4 and A.5 yields the one to be modeled in VMS:

$$
\frac{\partial \overline{\mathbf{u}}}{\partial t}+(\overline{\mathbf{u}} \cdot \nabla) \overline{\mathbf{u}}-\nu \nabla^{2} \overline{\mathbf{u}}+\rho^{-1} \nabla \bar{p}=-\nabla \cdot \hat{\tau}-\nabla \cdot \tau^{\prime}
$$

In this paper, only $\tau^{\prime}$ is modeled being neglected $\hat{\tau}$. This approach, which is the small-small strategy proposed by Hughes et al. [17] has been successfully used before in the context of finite volume techniques by Sagaut and Ciardi [33] and by Lehmkuhl et al. [22].

A suitable model should be used for the small-scale part of the subgrid scale tensor, i.e. $\tau^{\prime}$. Here, the WALE model [16] is used to close the formulation.

$$
\begin{aligned}
& \tau^{\prime}=-2 \nu_{S G S} \overline{S^{\prime}}+\left(\tau^{\prime}: \mathbf{I}\right) \mathbf{I} / 3 \\
& \nu_{S G S}=\left(c_{\text {wale }}^{\text {VMS }} l\right)^{2} \frac{\left(\overline{\mathcal{V}^{\prime}}: \overline{\mathcal{V}^{\prime}}\right)^{3 / 2}}{\left(\overline{S^{\prime}}: \overline{S^{\prime}}\right)^{5 / 2}+\left(\overline{\mathcal{V}^{\prime}}: \overline{\mathcal{V}^{\prime}}\right)^{5 / 4}} \\
& \overline{\mathcal{V}^{\prime}}=\frac{1}{2}\left(\nabla\left(\overline{\mathbf{u}^{\prime}}\right)^{2}+\nabla^{T}\left(\overline{\mathbf{u}^{\prime}}\right)^{2}\right)-\frac{1}{3}\left(\nabla\left(\overline{\mathbf{u}^{\prime}}\right)^{2}: \mathbf{I}\right) \mathbf{I}
\end{aligned}
$$


where $c_{\text {wale }}^{V M S}$ is the model constant, which is equivalent to the WALE constant for the VMS approach. Here, $c_{\text {wale }}^{V M S}=0.325$. 